Aus der Klinik für Allgemeine, Unfall- und Wiederherstellungschirurgie der Ludwig-Maximilians-Universität München

Direktor: Prof. Dr. med. Wolfgang Böcker

\title{
Osteoporose als Risikofaktor für das Versagen der winkelstabilen Plattenosteosynthese am proximalen Humerus
}

\author{
Dissertation \\ zum Erwerb des Doktorgrades der Medizin \\ an der Medizinischen Fakultät \\ der Ludwig-Maximilians-Universität zu München \\ vorgelegt von \\ Johannes Kindsvater \\ aus \\ Krumbach
}


Mit Genehmigung der Medizinischen Fakultät

der Ludwig-Maximilians-Universität München

Berichterstatter:

Mitberichterstatter:

Mitbetreuung durch den

promovierten Mitarbeiter:

Dekan:

Tag der mündlichen Prüfung:
Priv.-Doz. Dr. med. Florian Haasters

Prof. Dr. Matthias Pietschmann

PD Dr. Stefan Grote

Prof. Dr. Volkmar Jansson

Dr. med. Tobias Helfen

Prof. Dr. med. dent. Reinhard Hickel

21.06.2018 
1 Einleitung .............................................................................

1.1 Osteoporose und assoziierte Frakturen................................................... 5

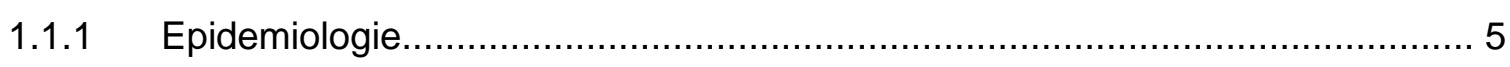

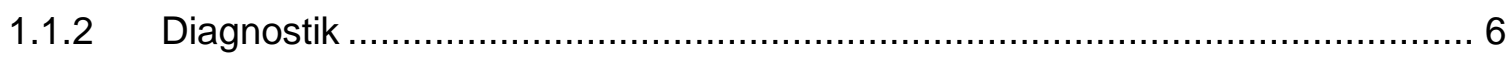

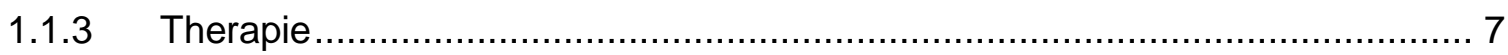

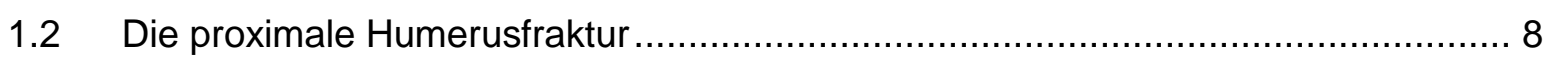

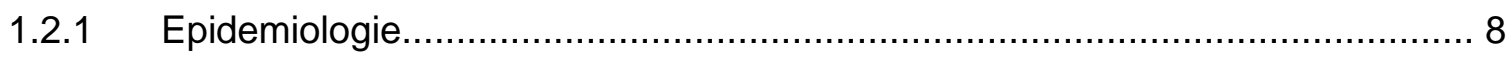

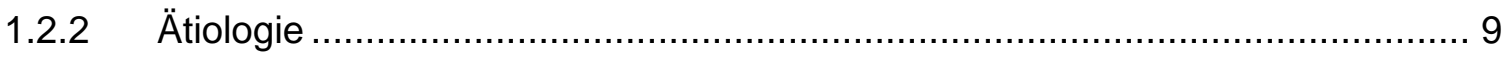

1.2.3 Frakturklassifikation........................................................................ 10

1.2.4 Therapie der proximalen Humerusfraktur ....................................................14

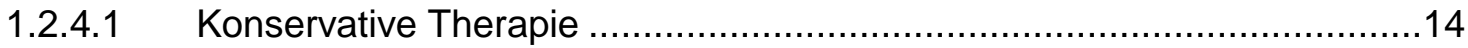

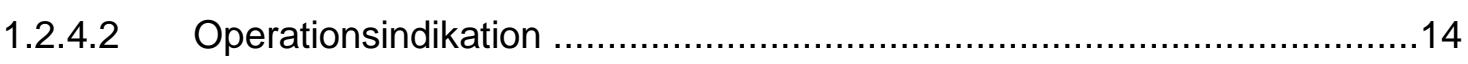

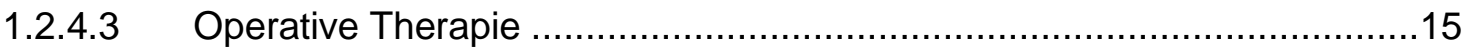

1.2.4.4 Komplikationen und Revisionsoperationen .......................................... 15

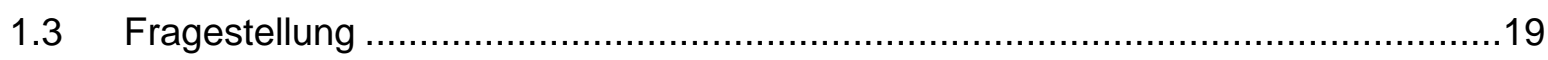

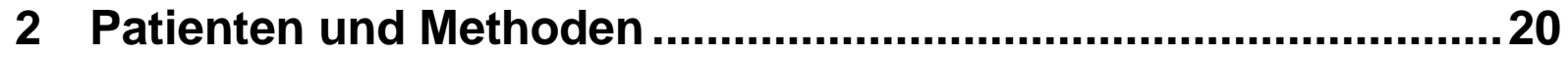

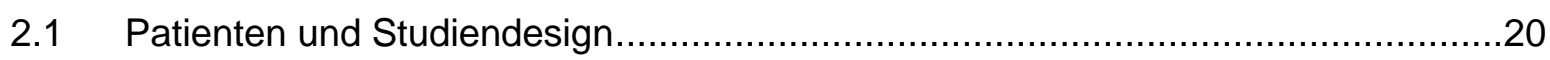

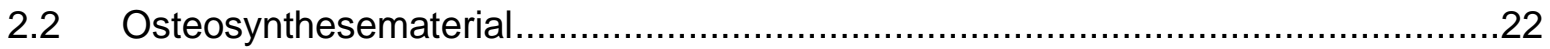

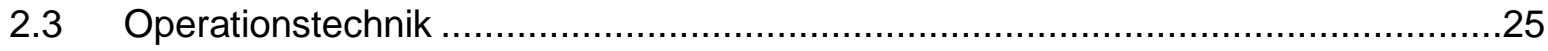

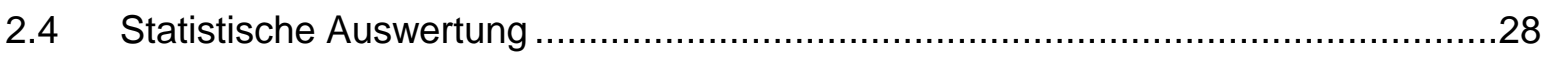

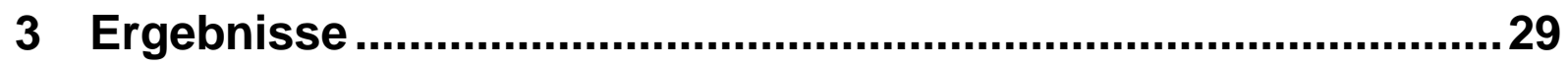

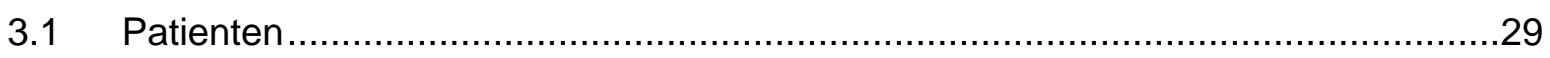

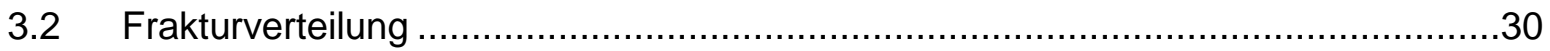

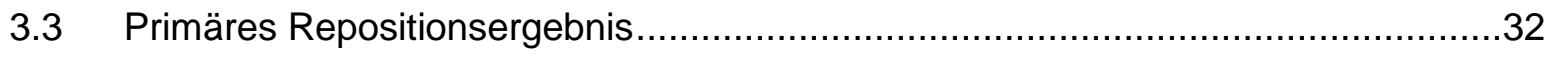

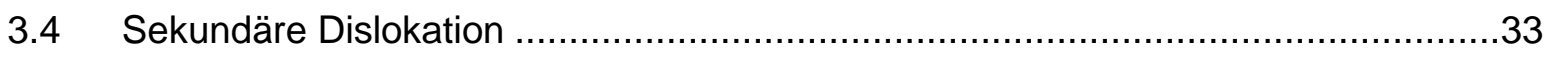

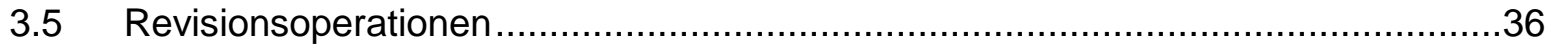

4 Diskussion ................................................................................. 40

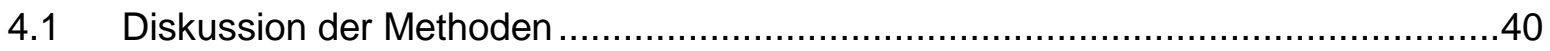




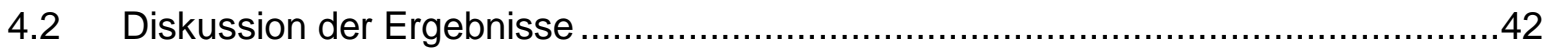

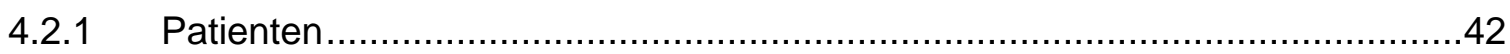

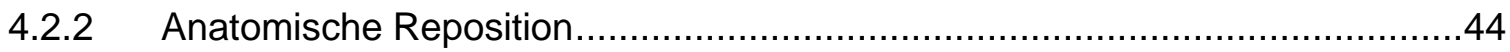

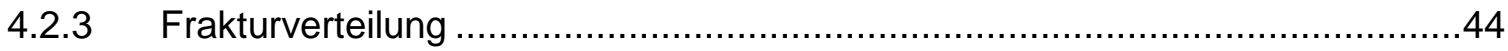

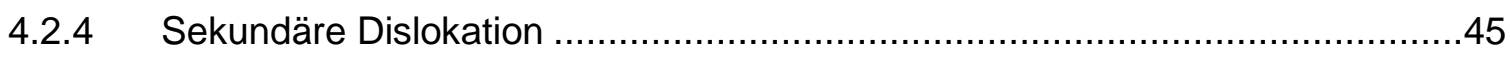

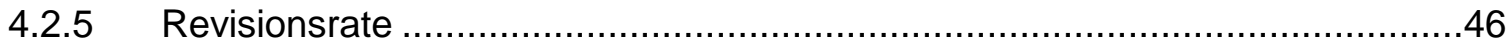

4.2.6 Sekundäre Dislokation und Revision bezogen auf den Frakturtyp ..................46

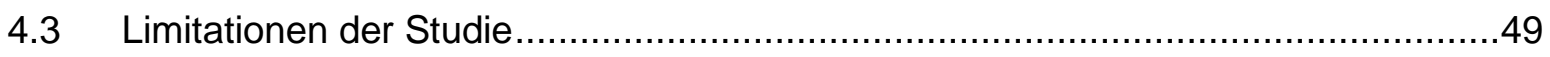

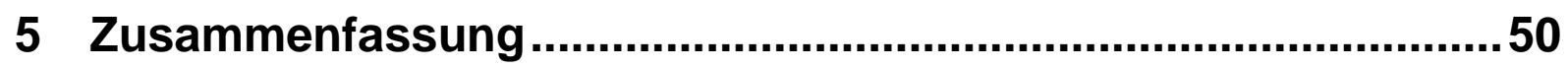

6 Literaturverzeichnis ............................................................... 52

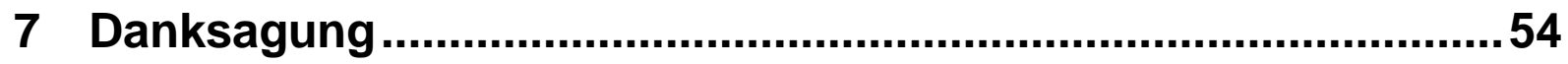




\section{Einleitung}

\subsection{Osteoporose und assoziierte Frakturen}

\subsubsection{Epidemiologie}

Nach der Empfehlung der World Health Organization (WHO) ist Osteoporose als Krankheit definiert, die durch eine Abnahme der Knochenmasse und Verschlechterung der Mikroarchitektur im Knochen zu einer erhöhten Brüchigkeit des Knochens und einem Anstieg des Frakturrisikos führt [1]. Osteoporose ist eine häufig unterdiagnostizierte und untertherapierte Krankheit von erheblicher gesellschaftlicher und gesundheitsökonomischer Relevanz [2, 3].

Die Krankheit zeigt eine stark geschlechts-, und altersabhängige Verteilung. Im Jahr 2009 waren in Deutschland bei einer Prävalenz von 14\% ab einem Alter über 50 Jahren, geschätzt 6,3 Millionen Menschen an Osteoporose erkrankt, darunter 5,2 Millionen Frauen [4]. Dies betraf jede vierte Frau und jeden siebzehnten Mann [4]. Für Patienten mit bereits aufgetretener Fraktur konnte unabhängig vom Geschlecht eine weitaus höhere Prävalenz von über 55\% nachgewiesen werden [2]. Die Inzidenz in Deutschland lag im Jahr 2009 bei 2,1\% (3,6\% Frauen und 0,9\% Männer), was 885000 Neuerkrankungen pro Jahr entspricht [4]. Dabei besteht mit steigendem Alter ein Trend zu einer höheren Inzidenz: So liegt die Inzidenz zwischen 50 und 54 Jahren bei 2,1\% für Frauen und 0,4\% für Männer und über einem Alter von 74 Jahren bei je 5,8\% und 2,3\% [4].

Die eigentlichen Einschränkungen der Lebensqualität für die Patienten und der Großteil der Kosten für das Gesundheitssystem entstehen mit dem Auftreten von Frakturen und ihren Folgezuständen [3]. Mehr als die Hälfte aller Betroffenen erleidet innerhalb von vier Jahren mindestens eine Fraktur, wovon 13\% die Schulter betreffen [4]. Die geschätzten direkten Kosten für die Behandlung von Osteoporose und nachfolgenden Frakturen machten in Deutschland im Jahr 2003 etwa 5,4 Milliarden Euro aus, wobei über 50\% auf stationäre Behandlungen zurückzuführen sind [3]. Die am häufigsten mit Osteoporose assoziierten Frakturen sind proximale Femurfrakturen, distale Radiusfrakturen, proximale Humerusfrakturen und Wirbelkörperfrakturen [3]. Haasters et al. konnten zeigen, dass bei Patienten, die aufgrund einer dieser Frakturen stationär behandelt werden müssen die Prävalenz einer Osteoporose 56\% bei Frauen über 50 Jahren bzw. 59\% bei Männern über 60 Jahren beträgt [2]. Besteht der Verdacht auf Osteoporose wird die frühzeitige diagnostische Abklärung und gegebenenfalls das Einleiten geeigneter Therapiemaßnahmen 
empfohlen [5]. Insbesondere bei osteoporotischen Frakturen des proximalen Humerus bleibt in der klinischen Realität jedoch häufig eine entsprechende Diagnostik aus [2].

\subsubsection{Diagnostik}

Eine Basisdiagnostik, bestehend aus Anamnese, körperlicher Untersuchung, Knochendichtemessung, Röntgen oder alternativer Bildgebung und Labor, wird empfohlen, wenn das auf Grundlage der derzeit verfügbaren epidemiologischen Daten berechnete 10Jahresrisiko für Wirbelkörper-, und proximale Femurfrakturen 20\% übersteigt [5]. Zur Bestimmung der Knochendichte (BMD, engl. bone mineral density) und Diagnose einer Osteoporose existieren verschiedene Methoden [1]. Als Goldstandard hat sich die DXA (Dual X-Ray Absorptiometry) etabliert [1]. Hierbei kann durch die Differenz der Absorption zweier Röntgenstrahlen unterschiedlicher Energien die Knochendichte in $\mathrm{g} / \mathrm{cm}^{2}$ berechnet werden. Diese wird typischer Weise im Bereich des Oberschenkelhalses und der Lendenwirbel L1-L4 gemessen. Zur Beurteilung werden die Ergebnisse mit Werten gleichgeschlechtlicher, junger, knochenstoffwechselgesunder Menschen verglichen und die Abweichung in Standardabweichungen (SD) als sogenannter T-Wert angegeben. Dabei beschreibt ein T-Wert innerhalb einer Standardabweichung vom Vergleichskollektiv eine normale Knochendichte. Nach Definition der WHO gilt eine Abweichung des T-Werts von mindestens $-2,5$ Standardabweichungen als Osteoporose [1]. Bei einem Wert zwischen -2,5 und -1,0 Standardabweichungen spricht man von einer Osteopenie [1].

Neben der DXA als Goldstandard existieren weitere Verfahren zur Bestimmung der Knochendichte. So lässt sich mit der quantitativen Computertomografie (QCT) die Knochendichte für meist einen repräsentativen Knochenbereich (z.B. Unterarm) mit Hilfe von Schnittbildtechnik in $\mathrm{g} / \mathrm{cm}^{3}$ berechnen. Die QCT Messung ermöglicht die getrennte Betrachtung von Spongiosa und Kortikalis, verursacht aber häufig eine vergleichsweise hohe Strahlenbelastung [1]. 


\subsubsection{Therapie}

Der Dachverband Osteologie (DVO) empfiehlt als Basistherapie der Osteoporose eine tägliche medikamentöse Zufuhr von 1000 mg Kalzium und 800-1000 IE Vitamin D pro Tag, sofern dies nicht durch die Nahrung erfolgt [5]. Zusätzlich ist bei allen Patienten eine ausreichende Sturzprophylaxe und psychosoziale Betreuung essentiell, um das Sturzrisiko zu senken [5]. Die Indikationsstellung einer spezifischen medikamentösen Osteoporosetherapie erfolgt nach Risikoprofil in Abhängigkeit von Geschlecht, Alter, DXAKnochendichte und früheren Frakturen [5]. Generelle Therapieindikationen sind niedrigtraumatische Wirbelkörperfrakturen oder proximale Femurfrakturen bei einem T-Wert $<-2,0$, sowie eine bestehende oder geplante Therapie mit Glukokortikoiden mit einem Prednisolonäquivalent von 7,5 mg oder mehr täglich über einen Zeitraum von 3 Monaten, wenn der T-Wert unter 1,5 liegt oder bereits Frakturen aufgetreten sind [5]. Eine Senkung des Frakturrisikos konnte für Alendronat, Bazedoxifen, Denosumab, Ibandronat, Östrogene, Teriparatid, Raloxifen, Risedronat, Strontiumranelat und Zoledronat belegt werden [5]. Sind weitere Risikofaktoren vorhanden, kann bereits bei höheren T-Werten mit einer Therapie begonnen werden [5].

Obwohl adäquate Leitlinien zur Osteoporosebehandlung verfügbar sind, erhalten viele Osteoporosepatienten keine entsprechende Therapie. Im Jahr 2003 lag in Deutschland der Anteil der Osteoporosepatienten mit spezifischer Therapie bei lediglich 21,7\% [3]. 


\subsection{Die proximale Humerusfraktur}

\subsubsection{Epidemiologie}

Proximale Humerusfrakturen zählen mit einem Anteil von über $5 \%$ zu den häufigsten Frakturen des erwachsenen Menschen und stellen ab einem Alter von 60 Jahren nach proximalen Femur-, und distalen Radiusfrakturen die dritthäufigste Fraktur dar [6, 7]. In über $70 \%$ der Fälle sind Frauen nach dem 60. Lebensjahr betroffen [8, 9]. Bei einem Großteil der Patienten handelt es sich um aktive, sich selbst versorgende Menschen [10]. Nach Fraktur sind etwa $25 \%$ nicht mehr in der Lage unabhängig den Haushalt zu erledigen und $20 \%$ benötigen pflegerische Hilfe bei der hygienischen Versorgung [10]. Sechs Monate nach Fraktur leiden noch 67\% an Schmerzen, 57\% an einer eingeschränkten Schulterfunktion und 33\% klagen über Ängste oder Depressionen [11].

Während die Inzidenz für die Normalbevölkerung bei 63 pro 100000 Einwohner liegt [6], zeigt sich für Frauen ein exponentieller Anstieg der Inzidenz ab dem 40. Lebensjahr [8, 9, 12, 13]. Vergleicht man die Inzidenzen in den Altersgruppen von 60-69 und 70-79 Jahren, zeigt sich bei Frauen eine Zunahme von 200 auf 439 pro 100000 Einwohnern [12]. Durch den demographischen Wandel mit der einhergehenden Zunahme des Anteils älterer Menschen in der Bevölkerung, steigt auch die Zahl der pro Jahr auftretenden proximalen Humerusfrakturen [14]. Für die finnische Bevölkerung konnte zwischen 1970 und 1995 ein massiver Anstieg der altersspezifische Inzidenz von über 10\% pro Jahr beobachtet werden [14]. Nach 1995 blieb die altersspezifische Inzidenz weitgehen unverändert, was durch bessere Präventionsmaßnahmen von Stürzen und die medikamentöse Prophylaxe von Osteoporose assoziierten Frakturen erklärt werden könnte [15]. Dennoch nimmt die Zahl der notwendigen operativen Eingriffe zu [16]. Die steigende Anzahl der Frakturen und in der Folge notwendige Operationen stellt eine Last für das Gesundheitssystem dar. Im Jahr 2000 belief sich der Median der stationären Kosten einer proximalen Humerusfraktur in Frankreich auf 3786 Euro, bei einer Gesamtzahl von 12262 Fällen [17]. 


\subsection{2 Ätiologie}

In 89\% aller proximalen Humerusfrakturen ist ein leichtes Trauma, meist ein Sturz aus dem Stand, ursächlich [8]. Kristiansen et al. konnten zeigen, dass die Schwere des notwendigen Traumas bei Frauen ab einem Alter von 40 Jahren stark abnimmt [8]. Den Sturz auslösende Faktoren sind nach subjektiven Angaben von $72 \%$ der Patienten ein Stolpern oder Ausrutschen [18]. Der Sturzmechanismus kann bei $71 \%$ der Patienten durch ein direktes Trauma auf die Schulter oder den Oberarm nach Sturz auf die Seite, nach vorne oder schrägvorwärts beschrieben werden [18]. Im Gegensatz zu distalen Radiusfrakturen wird der Sturz häufig nicht vom Patienten abgefangen [18]. Als Folge des direkten Traumas lässt sich daher in 76\% der Patienten ein subkutanes Hämatom im Bereich der Schulter finden [18]. Aus einer Studie von Clement et al. mit 2335 Patienten über 65 Jahren geht hervor, dass etwa $35 \%$ aller Patienten mit multiplen Frakturen als Komponente eine proximale Humerusfraktur aufweisen. Circa 19\% aller Patienten mit proximaler Humerusfraktur hatten mindestens eine zusätzliche Fraktur. Dabei war die Kombination mit einer proximalen Femurfraktur oder einer distalen Radiusfraktur am häufigsten. Diese Studie lässt außerdem erkennen, dass bei Patienten über 65 Jahren für multiple Frakturen in über $80 \%$ der Fälle nur ein einfacher Sturz aus dem Stand ursächlich ist [19].

In einem von Roux et al. untersuchten Patientenkollektiv konnte bei etwa 34\% der Patienten mit proximaler Humerusfraktur eine Osteoporose nachgewiesen werden [20]. Weitere Risikofaktoren waren Sturz in der Vorgeschichte (33\%), verminderte körperliche Aktivität (30\%), Rauchen (28\%), Sehschwäche (26\%), Gangstörung (25\%), Schmerz in der unteren Extremität (19\%), Hörschwäche (16\%), Alkoholismus (15\%) und proximale Femurfraktur der Mutter (12\%) [20]. Außerdem fanden sich in diesem Kollektiv häufiger Diabetes mellitus, Epilepsie, Demenz und Depression als in der Normalbevölkerung [20]. 


\subsubsection{Frakturklassifikation}

In den letzten 80 Jahren wurden verschiedene Systeme zur Klassifikation der proximalen Humerusfraktur entwickelt, die sich hinsichtlich der Beschreibung der Frakturmorphologie und ihrer Alltagstauglichkeit unterscheiden und jeweils Vor- und Nachteile mit sich bringen [21-26].

1934 beschrieb Codman in der ersten Klassifikation eine Fraktur, bestehend aus vier Fragmenten: Kalottenfragment, Tuberculum majus, Tuberculum minus und Schaftfragment [21] (siehe Abb. 1). Verschiedene Kombinationen dieser Fragmente sind möglich.

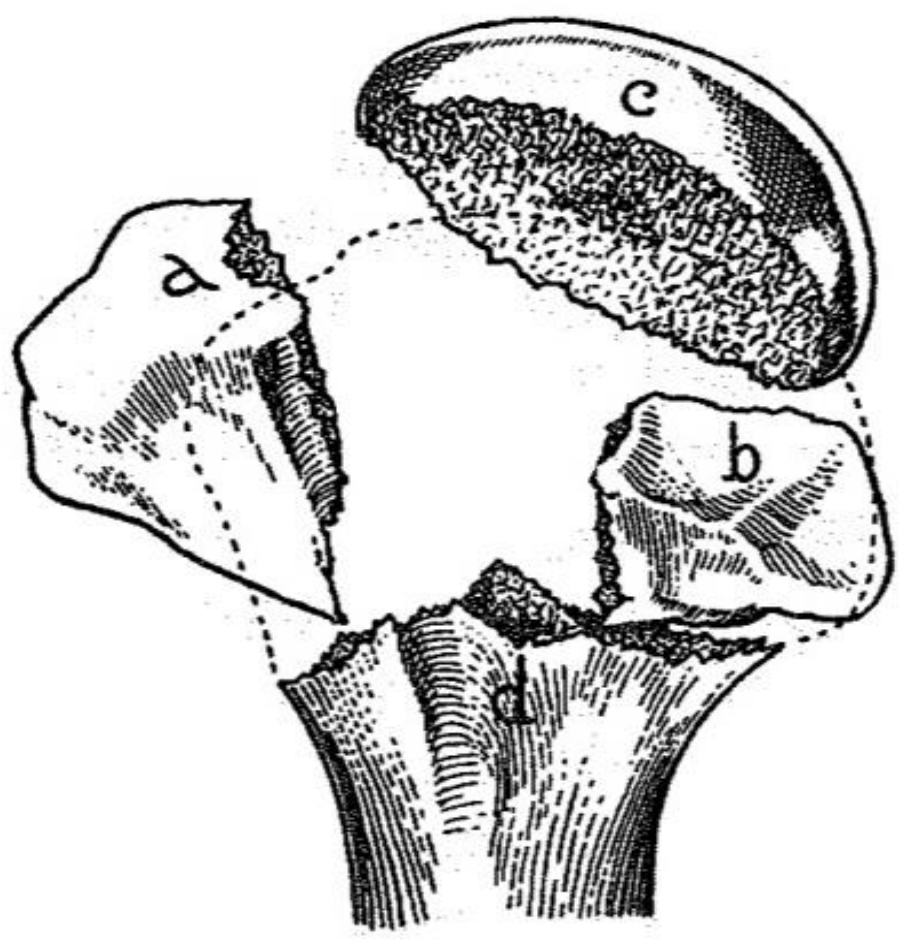

Abb. 1: Klassifikation der proximalen Humerusfraktur nach Codman [21]. a) Tuberculum majus b) Tuberculum minus c) Kalottenfragment d) Schaftfragment 
Die Klassifikation nach Neer aus dem Jahr 1970 basiert auf dem Dislokationsgrad der einzelnen Fragmente zueinander [22]. Als Dislokation wurde ein Versatz von mehr als $1,0 \mathrm{~cm}$, sowie eine Achsenfehlstellung größer $45^{\circ}$ definiert. Nach diesen Kriterien werden die Frakturen in die Gruppen I-VI nach Neer eingeteilt (siehe Tab. 1). Je nach Anzahl der Fragmente, ist eine Einteilung in 2-Fragment-, 3-Fragment- und 4-Fragmentfrakturen üblich. Nachteile der Klassifikation sind die Beschränkung auf die 4 Hauptfragmente und die fehlende Beschreibung der Art der Dislokation. Es wird nicht beurteilt, ob ein Fragment zur Körperachse hin (Varusfehlstellung) oder weg von dieser (Valgusfehlstellung) abgewinkelt ist und der Grad der Einstauchung (impaktiert, nicht impaktiert) wird wenig berücksichtigt [27]. Außerdem wird etwa die Hälfte aller Frakturen, ohne weitere Differenzierung, in der Gruppe I zusammengefasst [28].

\section{Neer-Klassifikation}

\begin{tabular}{|c|l|}
\hline I & Minimale Dislokation (Dislokation $\left.<1,0 \mathrm{~cm},<45^{\circ}\right)$ \\
\hline II & Dislokation der Gelenkfläche mit Fraktur im Collum anatomicum (2 Fragmente) \\
\hline III & Dislokation des Schafts mit Fraktur im Collum chirurgicum (2 Fragmente) \\
\hline IV & Dislokation des Tuberkulum majus (2 oder 3 Fragmente) \\
\hline V & Dislokation des Tuberkulum minus (2, 3 oder 4 Fragmente) \\
\hline VI & Luxationsfraktur (2, 3 oder 4 Fragmente) \\
\hline
\end{tabular}

Tab. 1: Klassifikation der proximalen Humerusfraktur nach Neer [22].

Eine weitere Einteilung bietet die AO-(Arbeitsgemeinschaft für Osteosynthesefragen) Klassifikation, die von der Orthopedic Trauma Association (OTA) als OTA-Klassifikation übernommen wurde $[23,29]$. Hierbei werden die Frakturen in die drei Gruppen A (unifokal, extraartikulär), B (bifokal, extraartikulär) und C (intraartikulär) eingeteilt. Für jede Gruppe existieren drei Typen, die jeweils in drei Formen unterteilt werden, sodass 27 mögliche Frakturformen beschrieben werden können (siehe Abb. 2). Valgische, varische, impaktierte und nicht impaktierte Frakturformen werden unterschieden. Die AO/OTA-Klassifikation der proximalen Humerusfraktur macht eine äußerst präzise Einteilung der Frakturen möglich, wird aber auf Grund ihrer Komplexität und der daraus resultierenden schlechten Alltagstauglichkeit kritisiert [25]. Ein gemeinsamer Nachteil der Neer-, und AO/OTA- 
Klassifikation ist die niedrige Reproduzierbarkeit bei mehrfacher Anwendung durch einzelne Untersucher bzw. zwischen mehreren Untersuchern untereinander (Intra,- und Interobserver Reliabilität) [25].

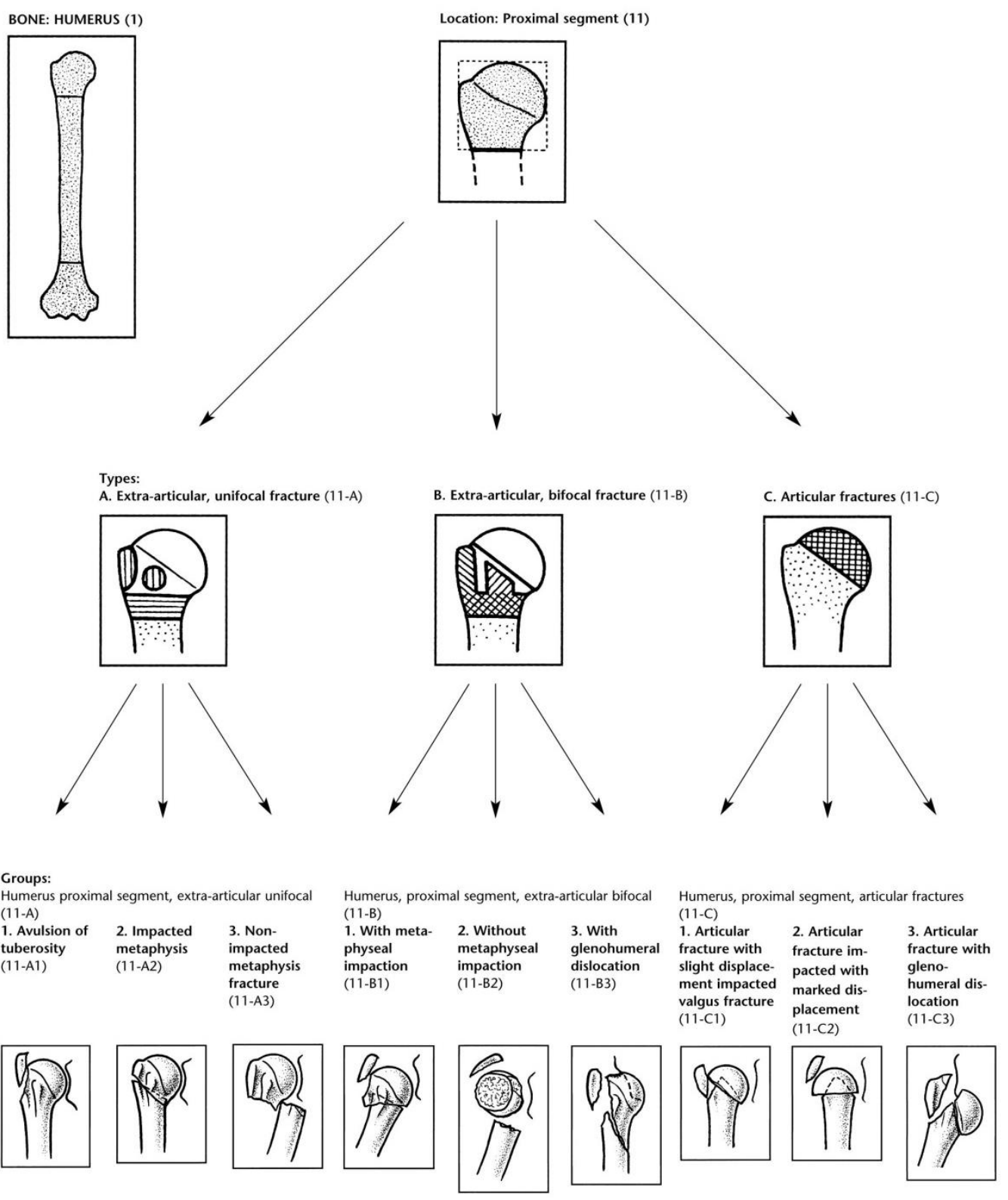

These fractures represent three part fractures, or fracture dislocations by the Neer classification. 
Die Klassifikation nach Hertel aus dem Jahr 2005 beruht auf der Codman Klassifikation ohne Berücksichtigung des Dislokationsgrads [24]. Durch ein binäres System, basierend auf 5 Fragen zum Frakturverlauf werden 12 mögliche Frakturtypen charakterisiert und durch zusätzliche Kriterien, wie den Versatz zwischen Schaft und Kopffragment (der sogenannte medial hinge) und die Länge des metaphysären posteromedialen Kopffragments ergänzt. Letztere scheinen einen Einfluss auf die Perfusion des Humeruskopfes zu haben [30].

Eine neuere Klassifikation nach Resch bezieht den Pathomechanismus und die Stellung des Kopffragments zum Humerusschaft in zwei Ebenen mit ein [25, 26]. Dadurch werden impaktierte und nicht impaktierte, sowie Varus-, und Valgusfrakturen sehr genau beschrieben und gleichzeitig eine vergleichsweise hohe Intra,- und Interobserver Reliabilität erreicht [25].

Da der Frakturtyp wegweisend für die Therapie ist und bestimmte Frakturtypen mit einer höheren Rate an Komplikationen einhergehen, ist eine möglichst genaue Beschreibung der Fraktur und Einteilung in ein Klassifikationssystem nötig [27, 31]. 


\subsubsection{Therapie der proximalen Humerusfraktur}

\subsubsection{Konservative Therapie}

Nicht dislozierte Frakturen machen etwa die Hälfte aller proximalen Humerusfrakturen aus und können in den meisten Fällen konservativ mit guten Ergebnissen behandelt werden [27, 28, 32]. Hierfür wird die Schulter beispielsweise für sieben Tage ruhiggestellt, danach mit Pendelübungen bewegt und anschließend passiv und zunehmend aktiv bewegt. Die zunehmende Belastung der Schulter erfolgt ab der 7. Woche [27]. Mehrere Studien konnten keinen Vorteil der operativen Therapie gegenüber der konservativen Therapie bezogen auf das funktionelle Ergebnis feststellen [33-35]. Die Rate der nachfolgenden Komplikationen und Revisionsoperationen ist unter konservativer Therapie jedoch bedeutend geringer, weshalb die Indikation zur Operation streng gestellt werden muss [32, 35]. Krettek et al. empfehlen ein konservatives Vorgehen für alle nicht dislozierten Frakturen unter 60 Jahren, und auch für alle dislozierten Frakturen bei einem Alter über 60 Jahren, sofern keine absolute Operationsindikation besteht (Luxationsfraktur, Head-Split-Fraktur, offener Weichteilschaden, pathologische Fraktur, nicht geschlossen reponierbare Schaftdislokation $>50 \%$ ) [32]. In die Therapieentscheidung sollten dabei stets der funktionelle Anspruch und Aktivität des Patienten, Komorbiditäten, Begleitverletzungen und das Alter mit einfließen [32].

\subsubsection{Operationsindikation}

Die Therapie der dislozierten Frakturen ist bei schlechter Evidenzlage umstritten [27, 32]. Kriterien zur Entscheidung zwischen konservativem oder operativem Vorgehen sind Dislokationsgrad, Komorbiditäten, Alter und Anspruch des Patienten [32]. Als gemeinhin anerkannte Kriterien für eine offene Reposition und innere Fixierung gelten die von Neer et al. 1970 publizierten Parameter: Dislokation der Tuberkula $>5 \mathrm{~mm}$, Schaftdislokation $>1 \mathrm{~cm}$, Achsabweichung des Kopffragments $>45^{\circ}$ [22]. Dennoch fehlt auch heute noch die wissenschaftliche Evidenz für die Überlegenheit der operativen Therapie gegenüber der konservativen Therapie [36]. Nach dem von Krettek et al. erstellten Therapiealgorithmus sind Luxationsfrakturen, Head-split-Frakturen, offene Frakturen, pathologische Frakturen und nicht reponierbare Frakturen klare Operationsindikationen. Alle anderen Patienten über 60 Jahre sollten unabhängig vom Dislokationsgrad konservativ behandelt werden [32]. Bei Patienten unter 60 Jahren werden Frakturen mit mehr als $1 \mathrm{~cm}$ Dislokation oder $45^{\circ}$ Fehlstellung operiert und bei geringerer Dislokation oder Fehlstellung die Entscheidung individuell getroffen [32].

Im Gegensatz dazu empfehlen Lill et al. die operative Therapie altersunabhängig sogar bei Tuberculum-majus-Frakturen mit Dislokation $\geq 2 \mathrm{~mm}$ oder knöchernem Rotatorenmanschettenausriss, Collum-chirurgicum-Frakturen mit Dislokation $>5 \mathrm{~mm}$ oder 
Abkippung der Kalotte $>20^{\circ}$, Frakturen mit metaphysärer Trümmerzone, Collumanatomicum-Frakturen, Gefäß-, und Nervenverletzungen und persistierender Instabilität nach Reposition [27].

\subsubsection{Operative Therapie}

Für die operative Versorgung proximaler Humerusfrakturen sind zahlreiche Verfahren verfügbar. Hierzu zählen die winkelstabile Plattenosteosynthese, die intramedulläre Marknagelung, die primäre Versorgung mit Hemiprothese oder inverser Prothese und minimalinvasive Verfahren wie perkutane Plattenosteosynthese, Humerusblock und Kirschner Draht (K-Draht) Osteosynthese [27, 32, 37-40]. Als etabliertes und am häufigsten verwendetes Verfahren gilt die winkelstabile Plattenosteosynthese [41-43]. Durch die winkelstabile Verankerung der Schraube in einem vorgeschnittenen Gewinde in der Platte wird ein höheres $\mathrm{Maß}$ an Primär-und Sekundärstabilität erreicht und die Gefahr der Schraubenlockerung gesenkt [44]. Dadurch ergeben sich Vorteile in der Therapie komplizierter Frakturen, insbesondere bei osteoporotischer Knochenlage [45], für die als alternatives Therapieverfahren oft nur der Gelenkersatz in Frage kommt [46].

\subsubsection{Komplikationen und Revisionsoperationen}

Die operativen Verfahren tragen im Vergleich zur konservativen Therapie ein höheres Risiko für Komplikationen und nachfolgende Revisionsoperationen [32]. Für die winkelstabile Plattenosteosynthese liegt die Komplikationsrate bei bis zu 48,8\% [47]. Die häufigsten Komplikationen sind sekundäre Dislokation (7-12,2\%), varische Abkippung des Kopffragments (16,3-25\%), avaskuläre Humeruskopfnekrose (4-10,8\%), Schraubenperforation in das Glenohumeralgelenk (Cut-Out) (7,5-23\%), subacromiales Impingement (2,9-4,8\%) und Infektion (1,9-3,5\%) [43, 47, 48]. Einige dieser Komplikationen treten oft zusammen auf oder bedingen sich gegenseitig. So führt die varische Abkippung und sekundäre Dislokation der Fragmente häufig zu einer konsekutiven Perforation der Schrauben [48]. In der Literatur wird der Begriff der sekundären Dislokation teilweise uneinheitlich angegeben. Ein Beispiel stellt der Vergleich von zwei großen Übersichtsarbeiten zum Thema dar: Während in der einen Studie für die sekundäre Dislokation eine Rate von 12,2\% angegeben wird [43], wird sie in der anderen Studie nicht als eigene Komplikation definiert, sondern durch andere Komplikationen umschrieben [47]. Die initiale anatomische Reposition der Fraktur scheint essentiell zu sein, um die Komplikations- und Revisionsrate zu senken [49].

Die Rate der nötigen Revisionsoperationen liegt für die winkelstabile Plattenosteosynthese bei $13-13,8 \%$ [43, 47, 48], wobei Schraubenperforation mit sekundärer Dislokation die 
Hauptgründe sind (19-31\%) [43, 47, 50]. Eine Dislokation der Fragmente oder des Osteosynthesematerials kann mit einer verminderten Knochenheilung oder Heilung in Fehlstellung einher gehen. Dadurch entstehen Funktionseinbußen und Beschwerden für den Patienten [51]. Bei einer Schraubenperforation ragen die Schrauben über den Gelenkknorpel des Humeruskopfs in das Glenohumeralgelenk hinein. Dies kann zu erheblichen Schäden des Knorpels und in Folge zu einer Arthrose führen. Revisionsoperationen umfassen die komplette oder teilweise Entfernung des Osteosynthesematerials, den erneuten Versuch einer Reposition (Reosteosynthese), arthroskopische Eingriffe zur Vergrößerung des subacromialen Raums oder als Ultima Ratio den Gelenkersatz mittels Endoprothese [52]. Im Folgenden sollen drei Fälle als anschauliche Beispiele dienen (siehe Abb. 3, 4, 5).

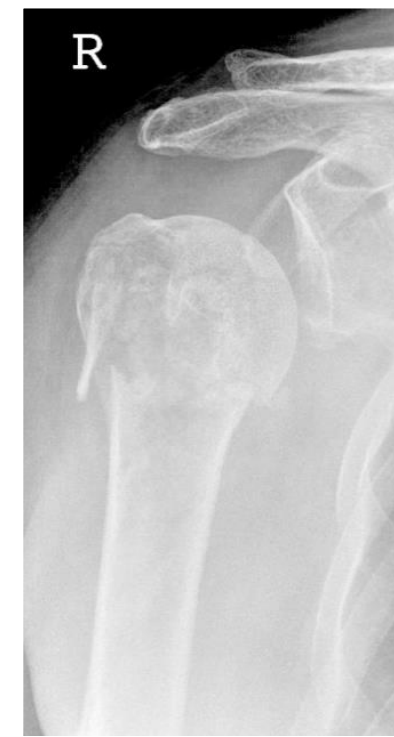

a

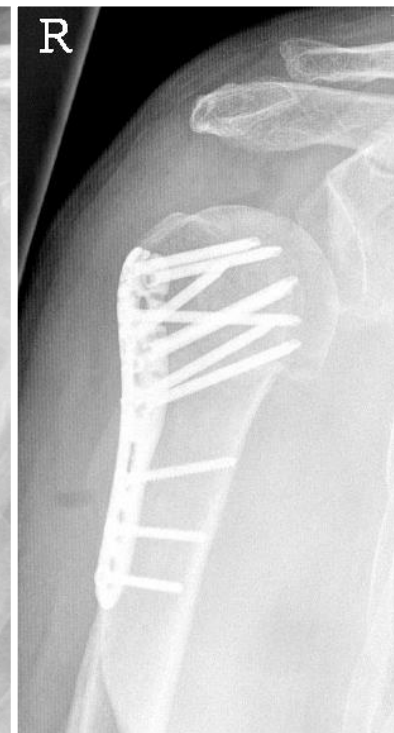

b

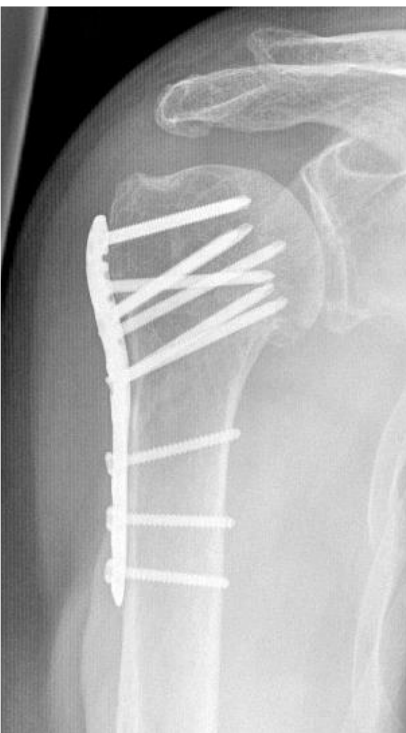

C

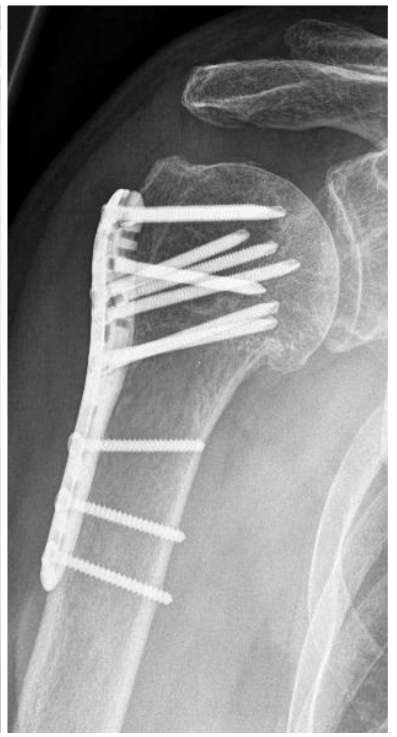

d

Abb. 3: Röntgenaufnahmen in True-a.p. Technik eines 75-jährigen Patienten mit proximaler Humerusfraktur. Beispiel eines komplikationslosen Verlaufs. a präoperatives Bild einer B1 Fraktur b postoperative Kontrolle nach winkelstabiler Plattenosteosynthese. Es besteht eine regelrechte Lage des Osteosynthesematerials mit anatomischer Reposition. c 8 Monate später zeigt sich eine deutliche Durchbauung des Frakturspalts bei regelrechter Schrauben-, und Plattenlage. d 2,5 Jahre postoperativ zeigt sich eine vollständige Konsolidierung der Fraktur bei regelrechter Schrauben-, und Plattenlage. Der Frakturspalt ist nicht mehr abzugrenzen. 


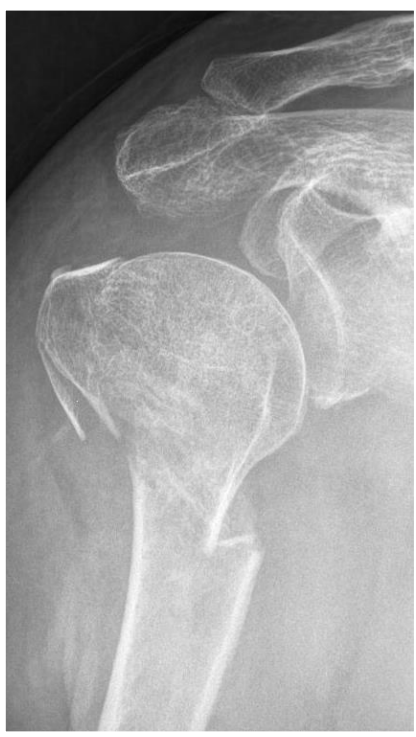

a

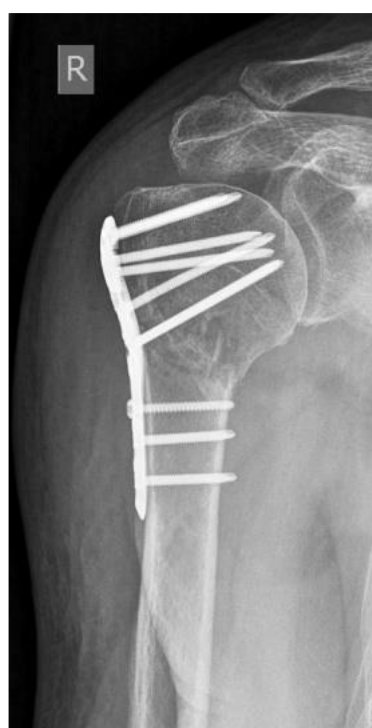

b

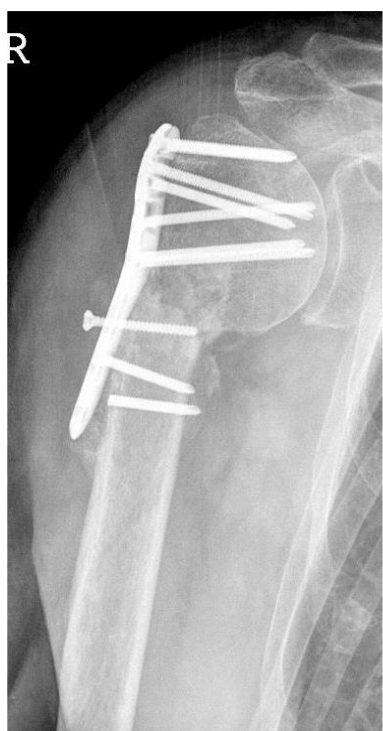

C

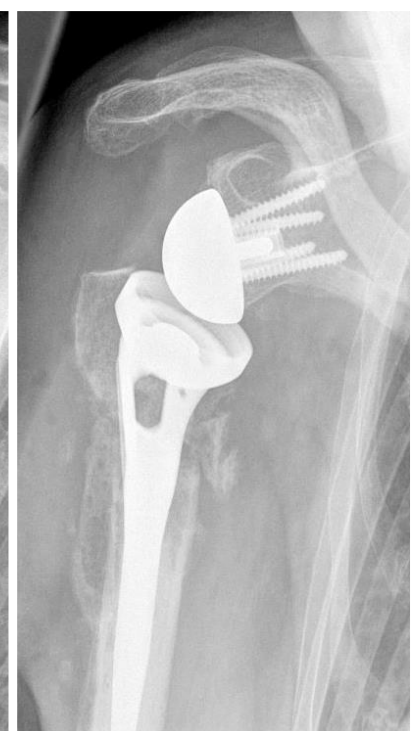

d

Abb. 4: Röntgenaufnahmen in True-a.p. Technik eines 77-jährigen Patienten mit proximaler Humerusfraktur. Osteopener Knochenaspekt. a präoperatives Bild einer B2 Fraktur b postoperative Kontrolle nach winkelstabiler Plattenosteosynthese. Es besteht eine regelrechte Lage des Osteosynthesematerials mit anatomischer Reposition. c 4,5 Monate später zeigt sich eine varische Abkippung des Kopffragments mit Ausreißen der Schaftschrauben. d Zustand nach Revisionsoperation mit Implantation einer inversen Prothese.

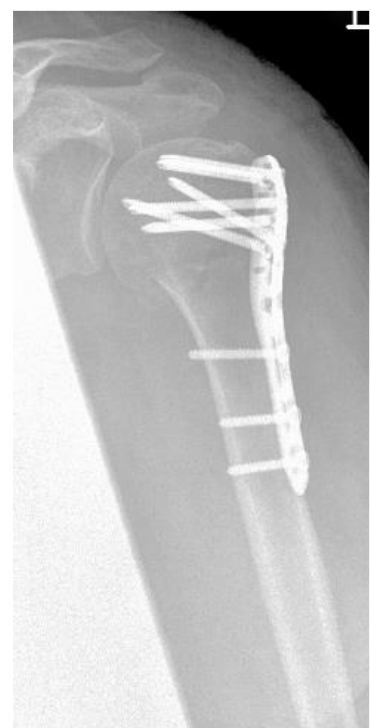

a

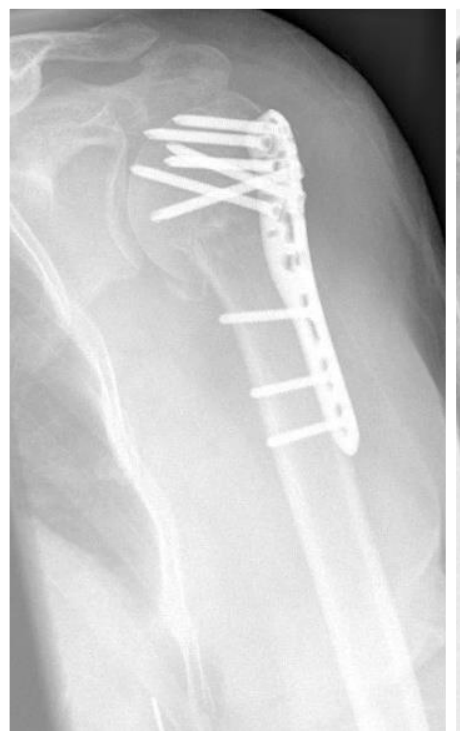

b

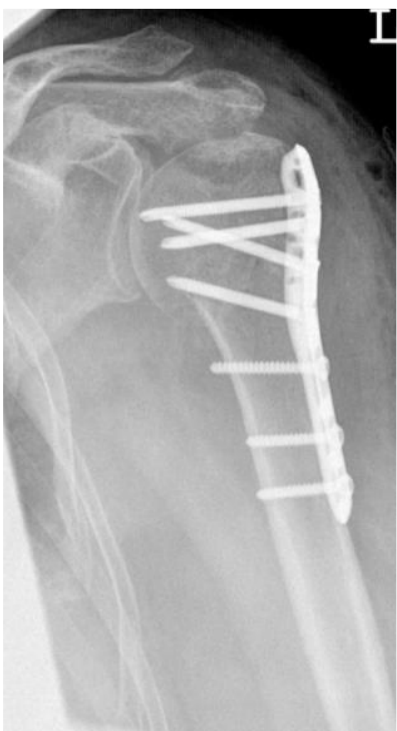

C

Abb. 5 Röntgenkontrolle in True-a.p. Technik nach operativer Behandlung einer 72-jährigen Patientin mit proximaler Humerusfraktur mit winkelstabiler Plattenosteosynthese. Osteopener Knochenaspekt. a postoperativ besteht eine regelrechte Lage des Osteosynthesematerials mit anatomischer Reposition. b 4 Wochen später zeigt sich eine varische Abkippung des Kopffragments mit Schraubenperforation in das Glenohumeralgelenk. c Zustand nach Revisionsoperation mit Entfernung der perforierenden Schrauben und Einbringung einer zusätzlichen Kalkarschraube.. 
Auf Grund des hohen Anteils an Komplikationen wurde oftmals versucht, Risikofaktoren für das Auftreten von Komplikationen zu identifizieren. Nach Erkenntnissen bisheriger Studien sind hohes Alter, Rauchen, fehlende anatomische Reposition, fehlende mediale Abstützung und bestimmte Frakturtypen Risikofaktoren für das Auftreten einer Komplikation [39, 48, 53]. Es konnte biomechanisch gezeigt werden, dass eine niedrige Knochendichte einen negativen Einfluss auf die stabile Verankerung des Osteosynthesematerials im Knochen hat [54]. In klinischen Studien konnte dies bisher nicht gezeigt werden [55]. Der Grund dafür ist die Heterogenität der Methoden zur Bestimmung der Knochendichte und der Verwendung des Begriffs Osteoporose in verschiedenen Studien [55]. Krappinger et al. konnten zeigen, dass Patienten mit einer im CT gemessenen erniedrigten Knochendichte im kontralateralen Humeruskopf ein erhöhtes Risiko für Komplikationen haben [39]. Diese Beobachtung konnte von Hepp et al. nicht bestätigt werden. Ferner handelt es sich nur um eine Korrelation von sekundärer Dislokation mit einer lokal gemessenen Knochendichte im proximalen Humerus und nicht um eine Korrelation mit dem Vorliegen einer nach internationalem Standard diagnostizierten Osteoporose mittels DXA [56]. 


\subsection{Fragestellung}

Angesichts der in der Literatur beschriebenen hohen Komplikationsraten nach winkelstabiler Plattenosteosynthese bei der proximalen Humerusfraktur, ist es von großer Wichtigkeit, relevante Risikofaktoren zu identifizieren und diese in die Therapiefindung miteinfließen zu lassen.

Ziel der vorliegenden Studie war es, den Einfluss der Osteoporose auf

- den Frakturtyp

- die Rate der sekundären Dislokation nach winkelstabiler Plattenosteosynthese

- die Rate und Art der nötigen Revisionsoperationen nach winkelstabiler Plattenosteosynthese

bei der proximalen Humerusfraktur zu untersuchen.

Bisher konnte nicht nachgewiesen werden, dass eine diagnostizierte Osteoporose die Rate an Komplikationen erhöht, was unter anderem an einer niedrigen Fallzahl und einer heterogenen Osteoporosediagnostik lag [55]. Daher war es in dieser Studie wichtig, die Osteoporosediagnostik nach internationalem Standard mittels DXA durchzuführen, um Reproduzierbarkeit zu schaffen.

Die Analyse der Frakturmorphologie ist entscheidend für die Therapie und Prognose. Patienteneigene Faktoren, die mit komplizierteren Frakturformen einhergehen, sollten daher identifiziert werden. Da durch eine verminderte Knochendichte die biomechanischen Eigenschaften des Knochens stark beeinflusst werden, ist eine Veränderung des Frakturverhaltens des Knochens naheliegend. Deshalb sollte in der Studie untersucht werden, inwiefern Osteoporose einen Einfluss auf die Frakturform hat.

Dadurch könnte in Zukunft für Risikopatienten eine alternative Therapie diskutiert werden, um Komplikationen und Revisionsoperationen zu vermeiden. Dies hätte sowohl positive Auswirkungen auf die Lebensqualität des Patienten, als auch auf die verursachenden Kosten der proximalen Humerusfraktur im Gesundheitssystem. 


\section{Patienten und Methoden}

\subsection{Patienten und Studiendesign}

Zwischen Januar 2002 und Dezember 2011 wurden an der chirurgischen Klinik Innenstadt der Ludwig-Maximilians-Universität München mit Hilfe einer elektronischen Datenbank (Microsoft Access) Patienten erfasst, die eine operative Versorgung einer proximalen Humerusfraktur mittels winkelstabiler Plattenosteosynthese erhalten hatten.

Zum Zeitpunkt der Aufnahme erhielten alle Patienten eine Röntgenuntersuchung der Schulter in zwei Ebenen (True a.p., und y-view Technik).

Die Fraktureinteilung erfolgte gemäß der AO/OTA-Klassifikation [23] unabhängig von zwei erfahrenen Schulterchirurgen. Hierbei beschränkte man die Einteilung auf die drei Hauptgruppen A, B, C sowie die Untergruppen 1, 2 und 3. Auf Grund der beschriebenen geringen Reproduzierbarkeit und Inter-, sowie Intraobserverreliabilität wurde auf die weitere Unterteilung in Subtypen verzichtet [57].

Bei eingeschränkter Beurteilbarkeit wurde zusätzlich eine Computertomografie (CT) der betroffenen Schulter angefertigt. Die Indikation zur Operation wurde anhand der NeerKriterien [22] für proximale Humerusfrakturen gestellt (Dislokation der Tuberkula $>5 \mathrm{~mm}$, Schaftdislokation $>1 \mathrm{~cm}$, Achsabweichung des Kopffragments $>45^{\circ}$ ). Präoperativ wurden die Patientendaten, der Frakturtyp nach AO/OTA-Klassifikation und die präoperativen Röntgenbilder in die Datenbank eingegeben. Postoperativ wurden erneut Röntgenaufnahmen angefertigt, um das primäre Repositionsergebnis zu beurteilen. Dazu wurden die jeweilige Lage der Platte und der Schrauben und die Reposition der Frakturfragmente evaluiert. Als anatomische Reposition wurde eine vollständige Wiederherstellung der medialen Abstützung bei gleichzeitiger Fragmentadaptation unter 2 $\mathrm{mm}$ Stufenbildung und unter $10^{\circ}$ Achsabweichung definiert. Die postoperativen Röntgenbilder und der Status des primären Repositionsergebnisses wurden in der Datenbank festgehalten.

Postoperativ erhielten die Patienten einen Gilchristverband zur Ruhigstellung, Analgetika zur Schmerztherapie und bis zur Mobilisation eine medikamentöse Thromboseprophylaxe. Während des stationären Aufenthalts erfolgte neben regelmäßigen Wundkontrollen die Durchführung einer Knochendichtemessung (DXA) gemäß den Leitlinien des Dachverbands Osteologie (DVO) [5]. Zur Bestimmung der Knochendichte wurde eine Dual-X-RayAbsorptiometrie (DXA) von Lendenwirbelsäule und proximalem Femur durchgeführt. Bei dem verwendeten Gerät handelte es sich um das Lunar Prodigy (GE Healthcare). Die Beurteilung wurde von zwei unabhängigen Radiologen gemäß der Leitlinien zur Anwendung für 
densitometrische Verfahren [58, 59] vorgenommen. Für jeden Patient wurde der individuelle Wert für die Knochendichte bestimmt und als T-Wert in die Datenbank eingetragen und für die Auswertung herangezogen. Vor Entlassung nach Hause wurde eine Röntgenkontrolle durchgeführt und mit den Patienten ein Termin in der Schultersprechstunde zur klinischen Verlaufskontrolle nach 6 Wochen vereinbart. Die Patienten wurden außerdem angehalten sich bei Zeichen eines Wundinfektes oder anderen Komplikationen sofort in der Notaufnahme vorzustellen.

Die Mobilisation erfolgte unter physiotherapeutischer Anleitung bereits während des stationären Aufenthalts und wurde anschließend ambulant fortgeführt. In der ersten Woche wurden lediglich Pendelübungen durchgeführt. Anschließend erfolgte für zwei Wochen eine passive Mobilisation und ab der vierten Woche die aktiv-assistierte Mobilisierung bis $90^{\circ}$ Abduktion. Der volle Bewegungsumfang war ab der 6 . Woche ohne Belastung erlaubt. Ab der 12. Woche konnte die Schulter wieder belastet werden.

Standardmäßig waren Kontrolluntersuchungen in der Schultersprechstunde nach 6 Wochen, 3, 12, und 24 Monaten postoperativ vorgesehen. Um eine langfristige Nachuntersuchung (Follow-Up) zu ermöglichen wurde den Patienten mindestens zwei Jahre nach Operation Einladungen zur Nachuntersuchung und zur Teilnahme an der Studie in Briefform zugeschickt. Falls keine Rückmeldung erfolgte, wurden die Patienten zwei Wochen später telefonisch kontaktiert. Patienten, von denen bereits ein Follow-Up von mindestens 24 Monaten vorhanden war, wurden nicht noch einmal zur Nachuntersuchung eingeladen. Die Nachuntersuchungen erfolgten im Rahmen der Schultersprechstunde unter fachärztlicher Aufsicht und beinhaltete eine Anamnese, körperliche Untersuchung und Röntgenaufnahmen in zwei Ebenen der jeweiligen Schulter. Bei Patienten, die nach mindestens 24 Monaten beschwerdefrei waren und bei denen das Osteosynthesematerial bereits entfernt worden war, wurden gemäß den Vorgaben der Ethikkommission keine weiteren Röntgenaufnahmen angefertigt. In der Anamnese wurde gezielt nach schulterabhängigen Beschwerden bei der Arbeit und im Altag, Schmerzen in Ruhe, bei Bewegung und in der Nacht, Bewegungseinschränkungen und Einschränkungen von Freizeitaktivitäten und sozialen Kontakten gefragt. In der klinischen Untersuchung der Schulter wurde das Bewegungsausmaß beidseits für Abduktion/Adduktion, Flexion/Extension, Außenrotation/Innenrotation erfasst und auf bewegungsabhängige Schmerzen geachtet.

Auf den Röntgenbildern wurden Schrauben,- und Plattenlage, sowie Stellung der Frakturfragmente analysiert. Dabei wurden postoperativ auftretende Fragmentdislokation $>2 \mathrm{~mm}$, Achsabweichung $>10^{\circ}$, Perforation (Cut-Out) der Schrauben in das Glenohumeralgelenk und Dislokation oder Bruch des Osteosynthesematerials als sekundäre Dislokation definiert. Die Rate der sekundären Dislokation wurde in der Datenbank festgehalten. 
Beim Auftreten von Komplikationen wurden mit den Patienten die möglichen Therapieoptionen besprochen und je nach Einzelfall therapiert. Alle sich daraus ergebenden Revisionsoperationen wurden registriert und der Revisionsgrund, sowie die Art der Revision in die Datenbank aufgenommen. Nicht berücksichtig wurden Revisionsoperationen auf Grund einer Komplikation im Weichteilbereich, sowie elektive Metallentfernungen, die auf Wunsch des Patienten oder wegen eines subacromialen Impingements durchgeführt wurden.

Einschlusskriterien für die vorliegende Studie waren eine operative Versorgung mit winkelstabiler Plattenosteosynthese, eine während des stationären Aufenthalts durchgeführte Knochendichtemessung mittels Dual-X-Ray-Absorptiometrie (DXA), ein Alter über 50 Jahre für Frauen und über 60 Jahre für Männer, sowie ein Follow-Up über mindestens 24 Monate nach Operation. Schwerwiegende neurologische und psychiatrische Erkrankungen, Incompliance oder Unvermögen an einer strukturierten Nachbehandlung teilzunehmen, maligne Grunderkrankung, andere Knochenstoffwechselerkrankungen als Osteoporose, offene Frakturen, vorbestehende Arthrose, pathologische Frakturen, initiale neurovaskuläre Verletzungen, Voroperationen an der verletzten Schulter, fehlender Versicherungsschutz, Ablehnung einer Osteoporosediagnostik, Versterben während des stationären Aufenthalts und fehlende Auswertbarkeit aus technischen Gründen wurden als Ausschlusskriterien definiert.

Alle Patientendaten wurden einer Pseudonymisierung unterzogen. Die Durchführung der Studie erfolgte gemäß den Richtlinien der Helsinki-Erklärung für Prinzipien medizinischer Forschung und den Empfehlungen der Good Clinical Practice [60] und wurde von der Ethikkommission (Nr. 156-12) der Ludwig-Maximilians-Universität München genehmigt.

\subsection{Osteosynthesematerial}

Für die winkelstabile Plattenosteosynthese wurde in großer Mehrheit die Philos-Platte der Firma Synthes GmbH (Oberdorf, Schweiz) verwendet. In wenigen Einzelfälle kam ein vergleichbares winkelstabiles Implantat zum Einsatz (Non Contact Bridging Proximal Humerus (NCB-PH) Platte, Firma Zimmer (USA)). Die Form der Platte ähnelt der Oberfläche des proximalen Humerus, auf dem die Platte implantiert wird (anatomisch präkonfektionierte Implantate). Bei der Philos-Platte enthielt der proximale Teil der Platte neun winkelstabile, unidirektionale Schraubenlöcher, die in die Reihen A bis E eingeteilt werden. Die Löcher unterschieden sich in der Anordnung auf der Platte und in der Ausrichtung, welche die Schraubenlage vorgab (siehe Abb. 6). Sie waren in Reihe A parallel angeordnet und leicht 
nach oben gerichtet, in Reihe B leicht vertikal versetzt und konvergent ausgerichtet, in Reihe C parallel angeordnet und aufwärts divergierend ausgerichtet. Reihe D beinhaltete ein LCP (Locking compression plate) - Kombinationsloch, mit leichter Ausrichtung nach oben. Das Kombinationsloch war für den Besatz von Schrauben mit oder ohne Winkelstabilität geeignet. In Reihe E befanden sich zwei leicht versetzte Schraubenlöcher, die ebenfalls aufwärts divergierend ausgerichtet waren und Schrauben in die gegenüberliegende Kalkarregion platzieren ließen.

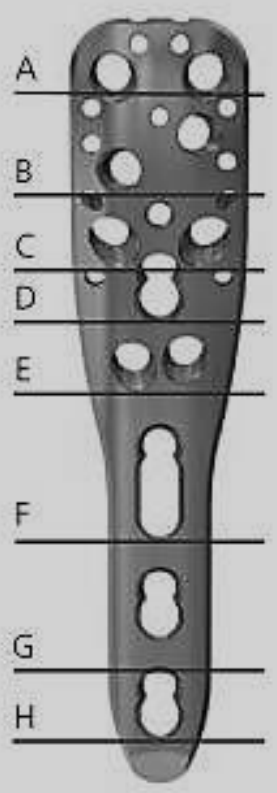

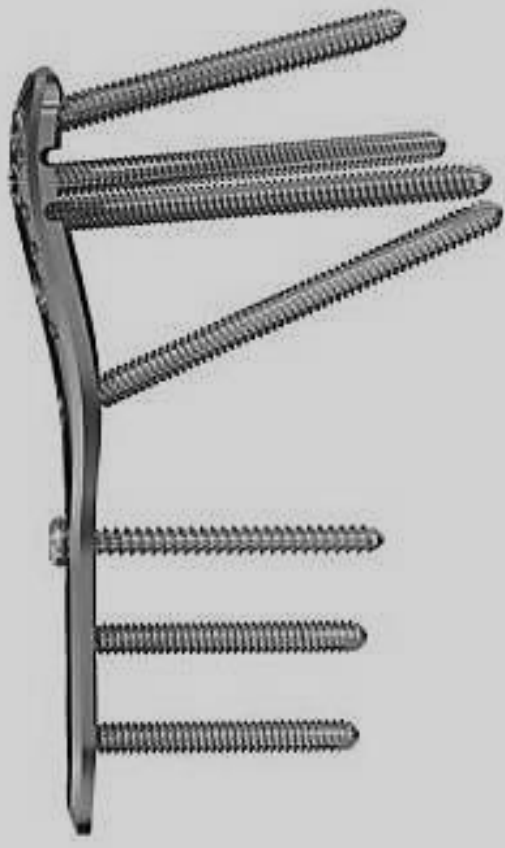

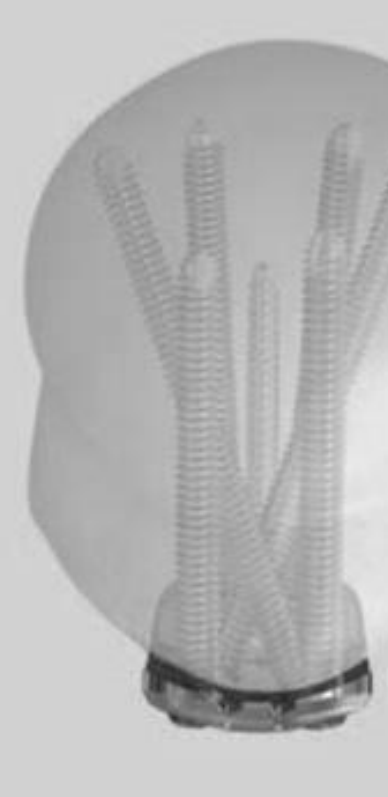

Abb. 6: a frontale Ansicht auf die Philos Platte (mit freundlicher Genehmigung der Firma Synthes). Neben den Schraubenebenen A-F sind die kleineren Löcher für die Cerclagen der Rotatorenmanschette zu sehen. b seitliche Ansicht der Platte mit Besatz durch winkelstabile Schrauben und eine Kortikalisschraube im Langloch. Die vertikale Ausrichtung der Schrauben kommt zur Darstellung. c axiale Ansicht der Platte am Humerusmodell. Die horizontale Ausrichtung der Schrauben mit Lage im Humeruskopf kommt zur Darstellung.

In Abhängigkeit von der Fraktursituation konnten die Schrauben individuell besetzt werden. Durch 10 Löcher mit je 2 mm Breite war eine temporäre Fixierung mittels Kirschner-Drähten möglich oder konnte eine Cerclage zur Befestigung der Sehnen der Musculi supraspinatus, infraspinatus und subscapularis geschlungen werden.

Der distale Teil der Platte enthielt drei LCP-Kombinationslöcher. Das am weitesten proximal gelegene Loch war länger und erlaubte somit eine vertikale Anpassung der Platte nach Besetzung mit einer Schraube.

Es konnten Kompressionsschrauben und winkelstabile Verriegelungsschrauben verwendet werden. Die Kompressionsschrauben hatten einen Gewindedurchmesser von 3,5 mm, einen Schraubenkerndurchmesser von 2,5 mm und einen Kopfdurchmesser von $6 \mathrm{~mm}$. Zum 
Einbringend der Schrauben wurden eine 3,5 mm Universal-Bohrbüchse, ein 2,5 mm Bohrer, ein Tiefenmessgerät und ein Sechskantschraubendreher benötigt.

Verriegelungsschrauben besaßen selbstschneidende, konische Köpfe, schneidende Spannuten und abgerundete Spitzen. Im Gegensatz zu den Kompressionsschrauben hatten Verriegelungsschrauben einen Schraubenkerndurchmesser von 2,8 mm. Für monokortikale Schrauben im Diaphysenbereich wurden auch selbstbohrende Schrauben mit nicht abgerundeter Spitze verwendet. Es wurden eine Bohrbüchse, ein 2,8 mm LCP-Bohrer mit Längenmarkierungen und ein Sechskantschraubendrehereinsatz mit 1,5 NM Drehmomentbegrenzer benötigt. Der Drehmomentbegrenzer sorgte für ein optimales Anzugsmoment der winkelstabilen Schrauben im Gewinde der Platte. 


\subsection{Operationstechnik}

Alle Patienten wurden nach erfolgter Aufklärung gemäß einem einheitlichen Operationsprotokoll operiert. Alle Operationen wurden von einem Facharzt für Orthopädie und Unfallchirurgie durchgeführt oder angeleitet. Zunächst wurde der Patient in Beach-ChairPosition gelagert (siehe Abb. 7).

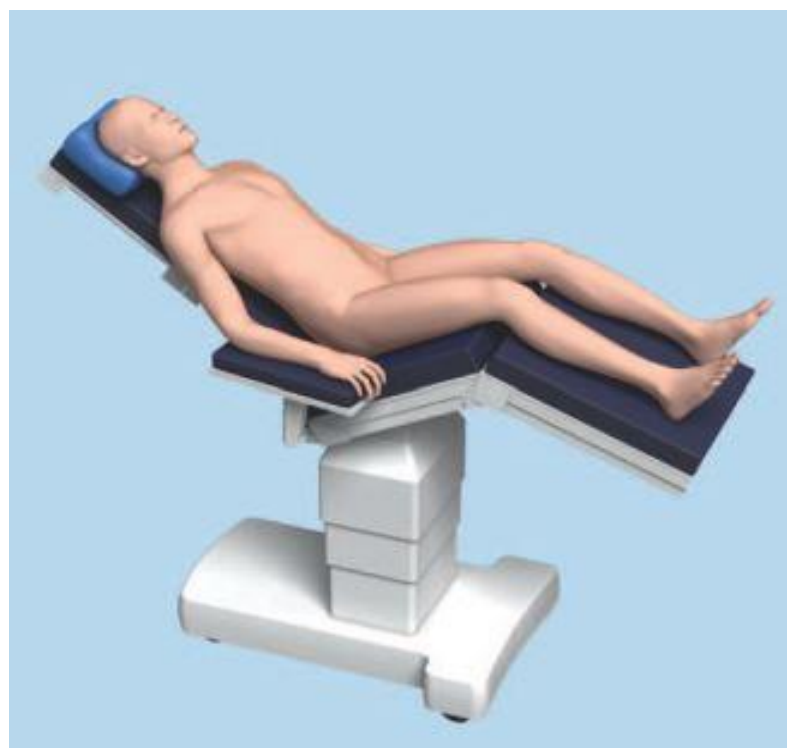

Abb. 7: Patientenlagerung in Beach-Chair-Position. Durch die Lagerung des Arms auf einem Seitentisch, wird der M. Deltoideus relaxiert (mit freundlicher Genehmigung der Firma Synthes).

Anschließend erfolgte das sterile Abwaschen. Bei jeder Operation wurde einmalig ein Antibiotikum intravenös verabreicht. Standardmäßig wurde der deltoideo-pektorale Zugang gewählt. Der ca. $10 \mathrm{~cm}$ lange Hautschnitt wurde nach distal leicht geschwungen zwischen Coracoid und Humerusschaft gesetzt (siehe Abb. 8).

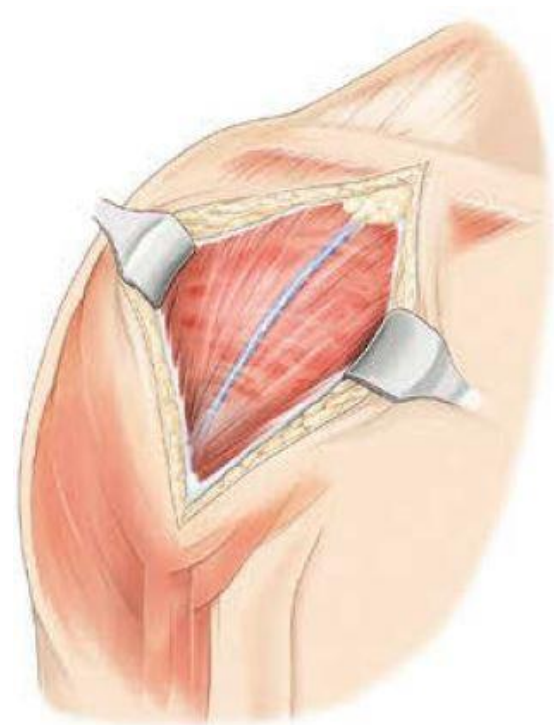

Abb. 8: Deltoideo-pektoraler Zugang (aus Lill H. Die proximale Humerusfraktur. Berlin: Springer; 2014) 
Es folgte die Präparation bis auf die Fascia clavipectoralis und Identifikation der V. cephalica, die für den weiteren Verlauf der Operation retrahiert und geschont wurde. Die Blutstillung wurde mit bipolarer Elektrokauterisation durchgeführt. Nach Inzision der Fascia clavipectoralis und Einsetzten eines U-Hakens mit Retraktion des M. deltoideus nach lateral, erfolgte die Darstellung des Humeruskopfs unter Schonung des N. axillaris. Bei bestehenden Läsionen der langen Bizepssehne erfolgten nun die Tenotomie am glenoidalen Ansatz und eine subpektorale Tenodese.

Die weiteren Schritte der Operationstechnik wurden hier zur besseren Anschaulichkeit am Modellknochen dargestellt. Abhängig von der Ausgangssituation und vom Frakturtyp wurden wenn möglich die Supraspinatus-, Subscapularis-, und Infraspinatussehne mittels Fibre-Wire 5.0 Fäden angeschlungen (siehe Abb. 9a). Anschließend wurden die Fibre-Wire Fäden durch die entsprechenden Löcher der PHILOS-Platte aufgefädelt, diese sodann aufgelegt und direkt lateral des Sulcus intertubercularis ausgerichtet (siehe Abb. 9b). Nachdem die Fraktur unter Sicht reponiert wurde, erfolgte die Verknotung der Cerclagen und die Fixierung der Platte mittels Kirschner-Drähten am Humeruskopf (siehe Abb. 9c).
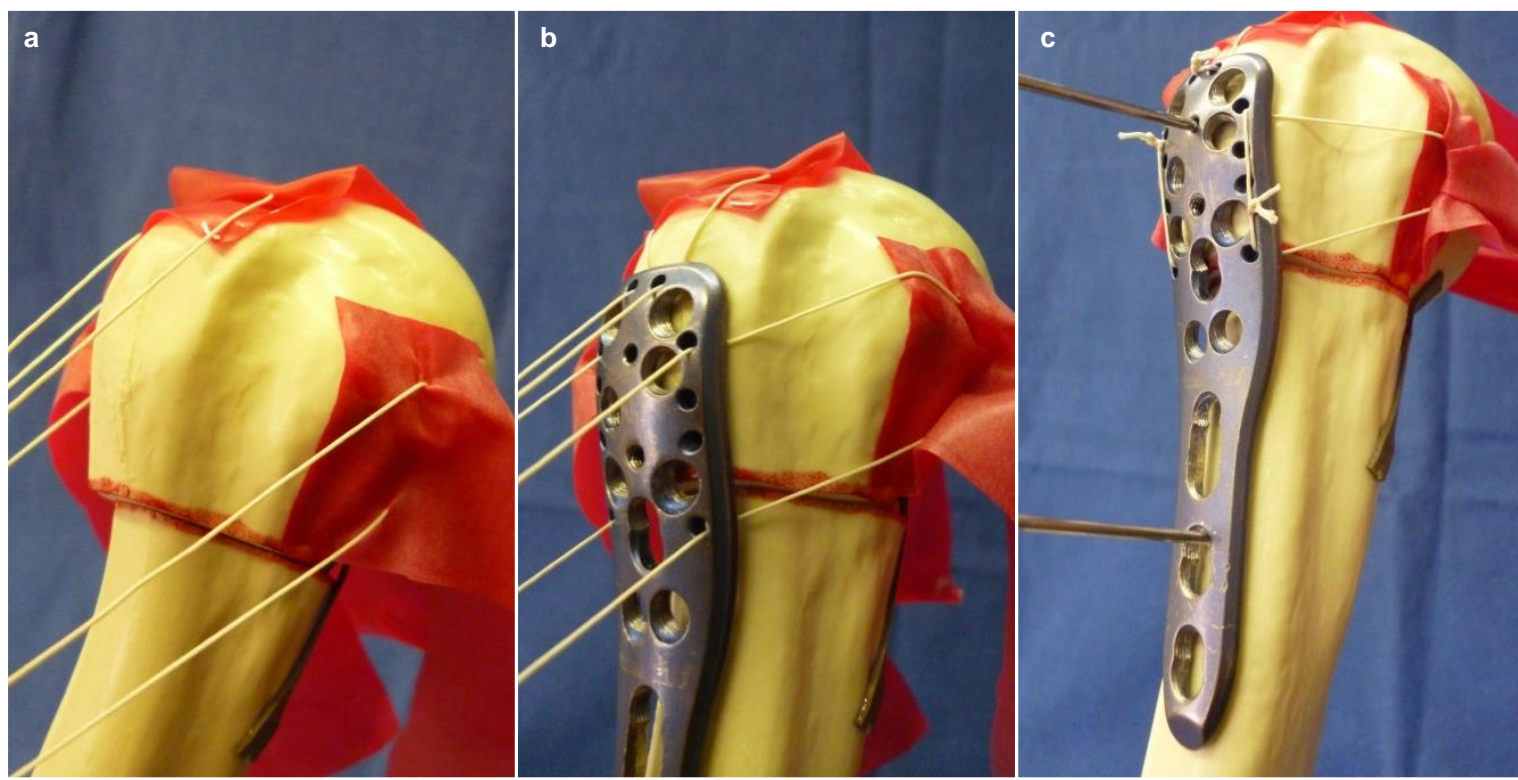

Abb. 9: Osteosynthese einer A3-Fraktur am Modellknochen. Simulation der Rotatorenmanschette durch ein rotes Gummiband. Die Frakturlinie wurde rot markiert a Anschlingen der Rotatorenmanschette mit Fibre-Wire 5.0 Fäden b Einfädeln und Platzieren der Platte c Fixierung der Platte mit Kirschnerdrähten und Cerclagen nach Reposition 


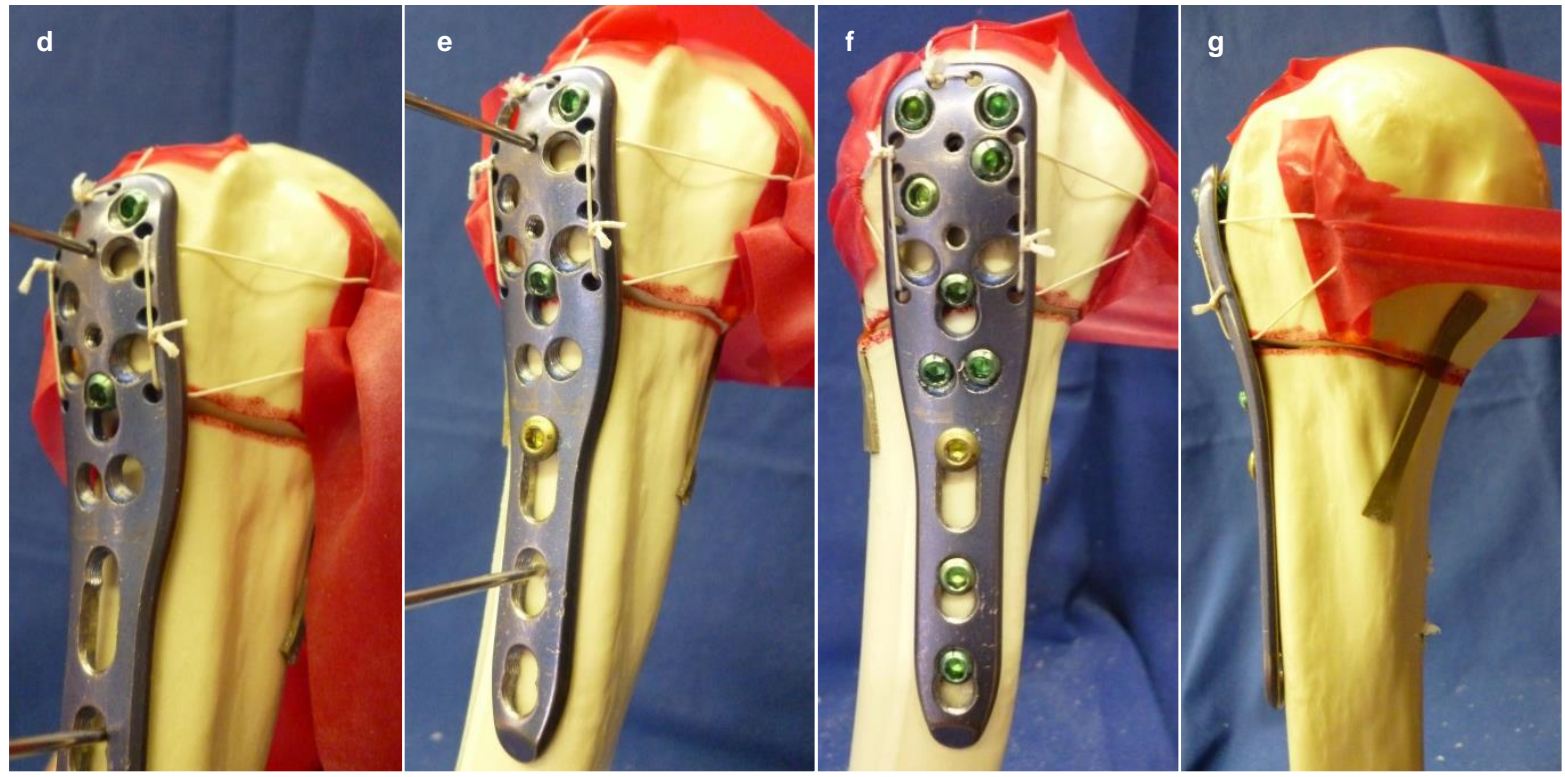

d Fixierung der Platte mit zwei winkelstabilen Schrauben im proximalen Teil (hier Reihe A und D) e Fixierung der Platte am Schaft durch Einbringen einer bikortikalen Kompressionsschraube im Langloch $\mathbf{f} \mathbf{u}$. $\mathbf{g}$ Endergebnis der Osteosynthese in zwei Ebenen. Die K-Drähte wurden entfernt und die Schraubenlöcher proximal und distal mit winkelstabilen Schrauben besetzt.

Die korrekte Lage der Platte, sowie die Reposition wurden dann mit dem Bildwandler kontrolliert und anschließend die zwei proximalen, winkelstabilen Schrauben eingedreht (siehe Abb. 9d). Im nächsten Schritt wurde eine bikortikale Schraube im Langloch platziert und die Platte damit an den Schaft herangezogen (siehe Abb. 9e). In einer erneuten Kontrolle mittels Bildwandler in 3 Ebenen wurden anatomische Reposition, Gelenkwinkel, Platten-, und Schraubenlage überprüft. Dann wurden zuerst die übrigen bikortikalen Schaftschrauben eingedreht und danach mehrere winkelstabile Schrauben im Humeruskopf. Die Anzahl der Schrauben im Kopf variierte in den Operationen, da die Belegung der Löcher von der individuellen Fraktursituation abhängt. Die oberste Schaftschraube wurde dann durch eine winkelstabile Schraube ersetzt. Im Anschluss wurden in einer erneuten Bildwandlerkontrolle die anatomische Reposition, Gelenkwinkel, Platten-, und Schraubenlage überprüft. Hierbei wurde insbesondere auf die subchondrale Schraubenlage der Schrauben im Humeruskopf geachtet. Zu diesem Zeitpunkt erfolgte außerdem eine klinische Funktionsprüfung der Schulter mit Kontrolle des Bewegungsausmaßes. Anschließend wurde die Wunde gespült und eine Redondrainage eingelegt. Danach folgte der Wundverschluss schichtweise, die Hautnaht fortlaufend und die Anlage eines sterilen Pflasterverbandes. 


\subsection{Statistische Auswertung}

Die statistische Auswertung erfolgte mittels Microsoft Excel 2010 und GraphPad Prism. Alle Daten wurden in einer Microsoft Access Datenbank dokumentiert und archiviert.

Für das Alter als kontinuierliche Variabel wurde der Mittelwert und die Standardabweichung berechnet. Als Testverfahren diente der t-Test für unabhängige, kontinuierliche Variablen. Als Varianzanalyse bei Vorliegen mehrerer Zielvariablen wurde eine ANOVA (analysis of variance) Testung durchgeführt.

Für kategorische Variablen wurden die Absolut-, und Prozentwerte berechnet. Für die folgenden Parameter wurde der Chi-Quadrat-Test für kategorische Variablen verwendet:

- Geschlecht

- Osteoporoseprävalenz

- Frakturverteilung

- Primäres Repositionsergebnis

- Rate sekundärer Dislokationen

- Rate an Revisionsoperationen

Bei Fallzahlen unter 5 wurde der Fisher Exact Test verwendet.

Für alle Berechnungen wurde ein Signifikanzniveau von $\leq 0,05$ festgelegt. 


\section{Ergebnisse}

\subsection{Patienten}

Unter allen Patienten, die nach proximaler Humerusfraktur mit winkelstabiler Plattenosteosynthese versorgt wurden, erfüllten 213 Patienten die Einschlusskriterien. Für 23 Patienten konnte keine Nachuntersuchung über 24 Monate durchgeführt werden. Davon waren 10 Patienten verstorben, 9 Patienten konnten wegen schlechtem Gesundheitszustand nicht an der Untersuchung teilnehmen und 4 Patienten waren zu einer unbekannten Adresse verzogen. Insgesamt konnte für 190 Patienten (89,2\%) ein Follow-Up über mindestens 24 Monate erhoben werden.

Unter diesen 190 Patienten zeigte sich ein Durchschnittsalter von 74,3 $( \pm 9,9)$ Jahren. Dabei war das Durchschnittsalter beim Vorliegen einer Osteoporose mit 75,7 $( \pm 9,3)$ Jahren signifikant höher als bei Patienten ohne Osteoporose $(71,3( \pm 10,3)) \quad(p<0,01)$. Im Gesamtkollektiv waren 164 Patienten weiblich (86,3\%) und 26 männlich (13,7\%).

Anhand der durch die Knochendichtemessung erhobenen T-Werte wurden 127 Patienten in die Osteoporosegruppe und 63 Patienten in die Kontrollgruppe eingeteilt. Damit lag die Osteoporoseprävalenz im Gesamtkollektiv bei 66,8\%. Eine Osteoporose fand sich bei Frauen häufiger (67,7\%) als bei Männern (61,5\%) (siehe Abb. 10). Dabei war der Anteil der Männer mit $12,6 \%$ in der Osteoporosegruppe und 15,9\% in der Kontrollgruppe in beiden Gruppen deutlich niedriger als der der Frauen. Die geschlechtsspezifischen Unterschiede waren ohne statistische Signifikanz $(p=0,54)$.

\section{Osteoporoseprävalenz}

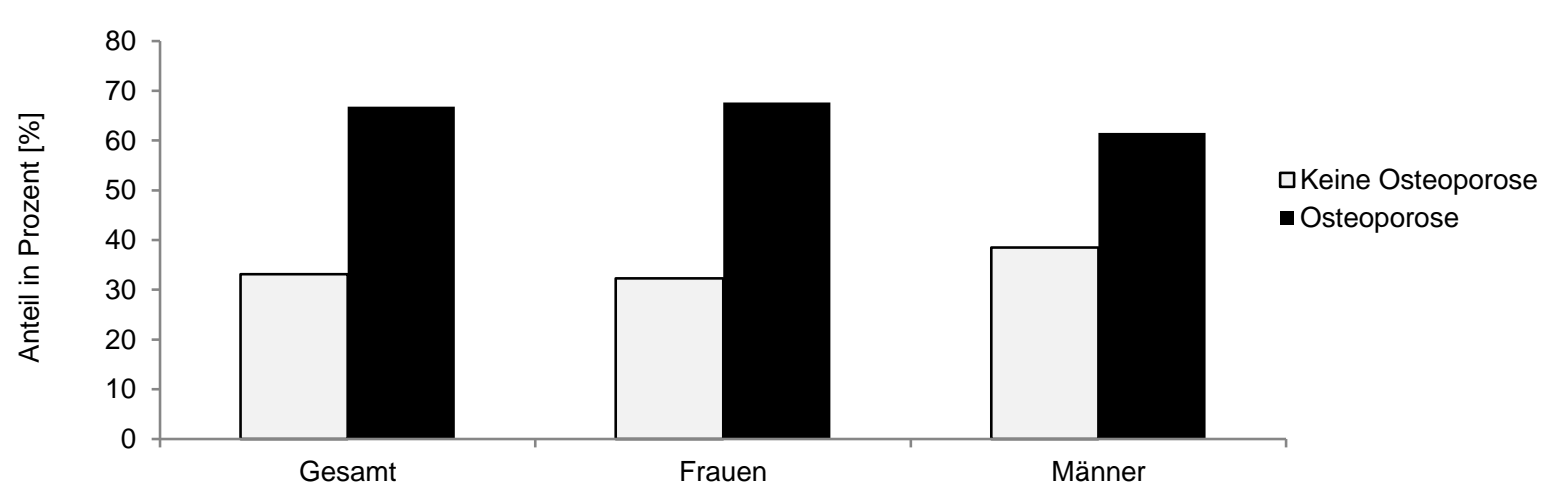

Abb. 10: Im Gesamtkollektiv bestand eine Osteoporoseprävalenz von $66,8 \%$. Bei Frauen zeigte sich mit $67,7 \%$ häufiger eine Osteoporose als bei Männern (61,5\%) ( $p=0,54)$. Die geschlechtsspezifischen Unterschiede waren ohne statistische Signifikanz. 


\subsection{Frakturverteilung}

Insgesamt wurden nach AO/OTA-Klassifikation 43 B2-Frakturen (22,6\%), 36 C2-Frakturen (18,9\%), 35 A3-Frakturen (18,4\%), 30 B1-Frakturen (15,8\%), 26 A2-Frakturen (13,7\%), 8 B3Frakturen (4,2\%), 6 C1-Frakturen (3,2\%), 4 C3-Frakturen (2,1\%) und 2 A1-Frakturen (1,1\%) registriert (siehe Abb. 11). Damit waren im Gesamtkollektiv B2-Frakturen mit 43 Fällen am häufigsten.

\section{Frakturverteilung im Gesamtkollektiv}

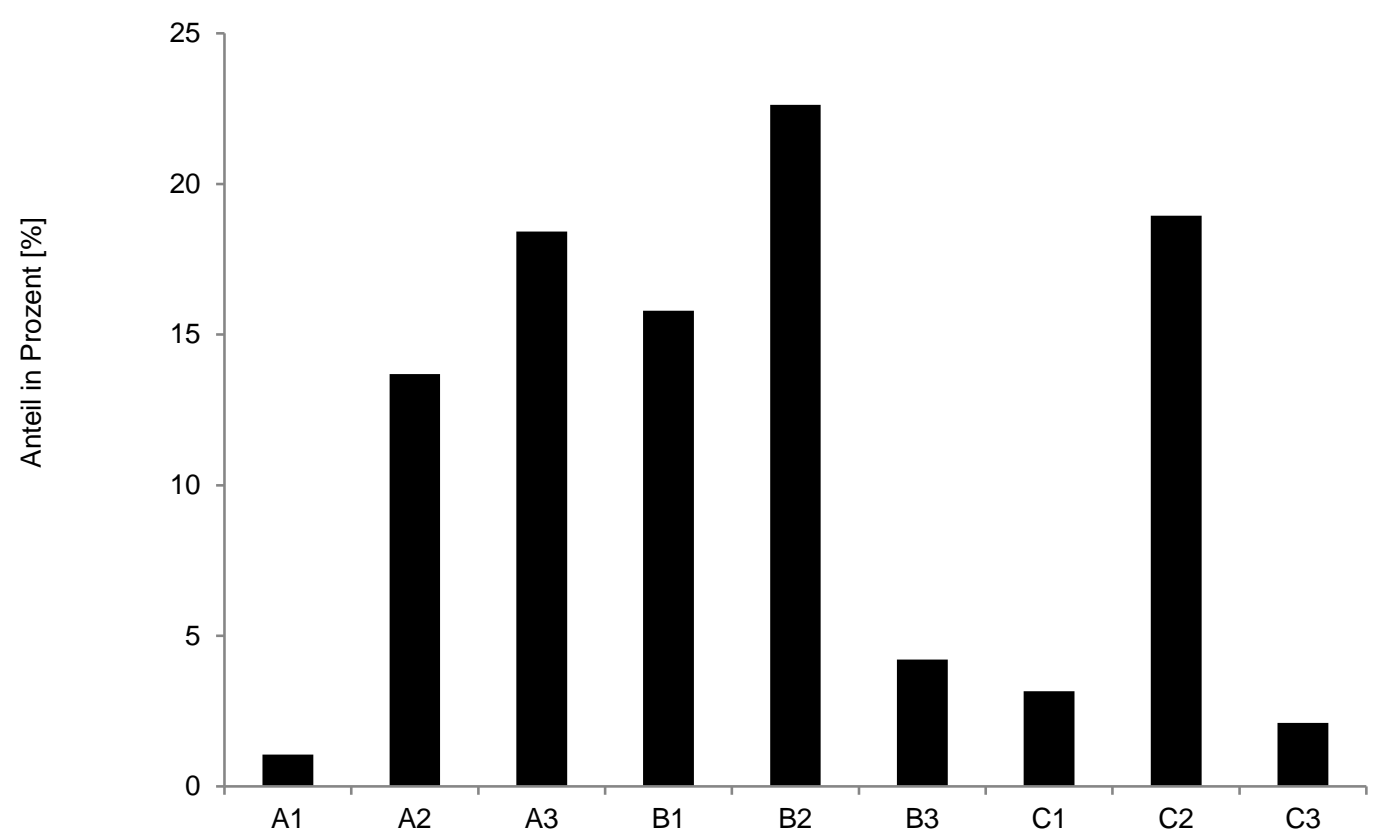

Abb. 11: B2-Frakturen waren mit 22,6\% insgesamt am häufigsten. C2: 18,9\%, A3: 18,4\%, B1: 15,8\%, A2: 13,7\%, B3: 4,2\%, C1: $3,2 \%$, C3: $2,1 \%, A 1: 1,1 \%$. 
Abbildung 12 gibt einen Überblick über die Frakturverteilung in beiden Gruppen: In der Osteoporosegruppe entsprach dies 33 B2-Frakturen, 27 C2-Frakturen, 24 A3-Frakturen, 18 A2-Frakturen, 16 B1-Frakturen, 4 C1-Frakturen, 2 B3-Frakturen, 2 A1-Frakturen und 1 C3Fraktur. In der Kontrollgruppe fanden sich 14 B1-Frakturen, 11 A3-Frakturen, 10 B2Frakturen, 9 C3-Frakturen, 8 A2-Frakturen, 6 B3-Frakturen, 3 C3-Frakturen und 2 C1Frakturen.

\section{Frakturverteilung der Gruppen}

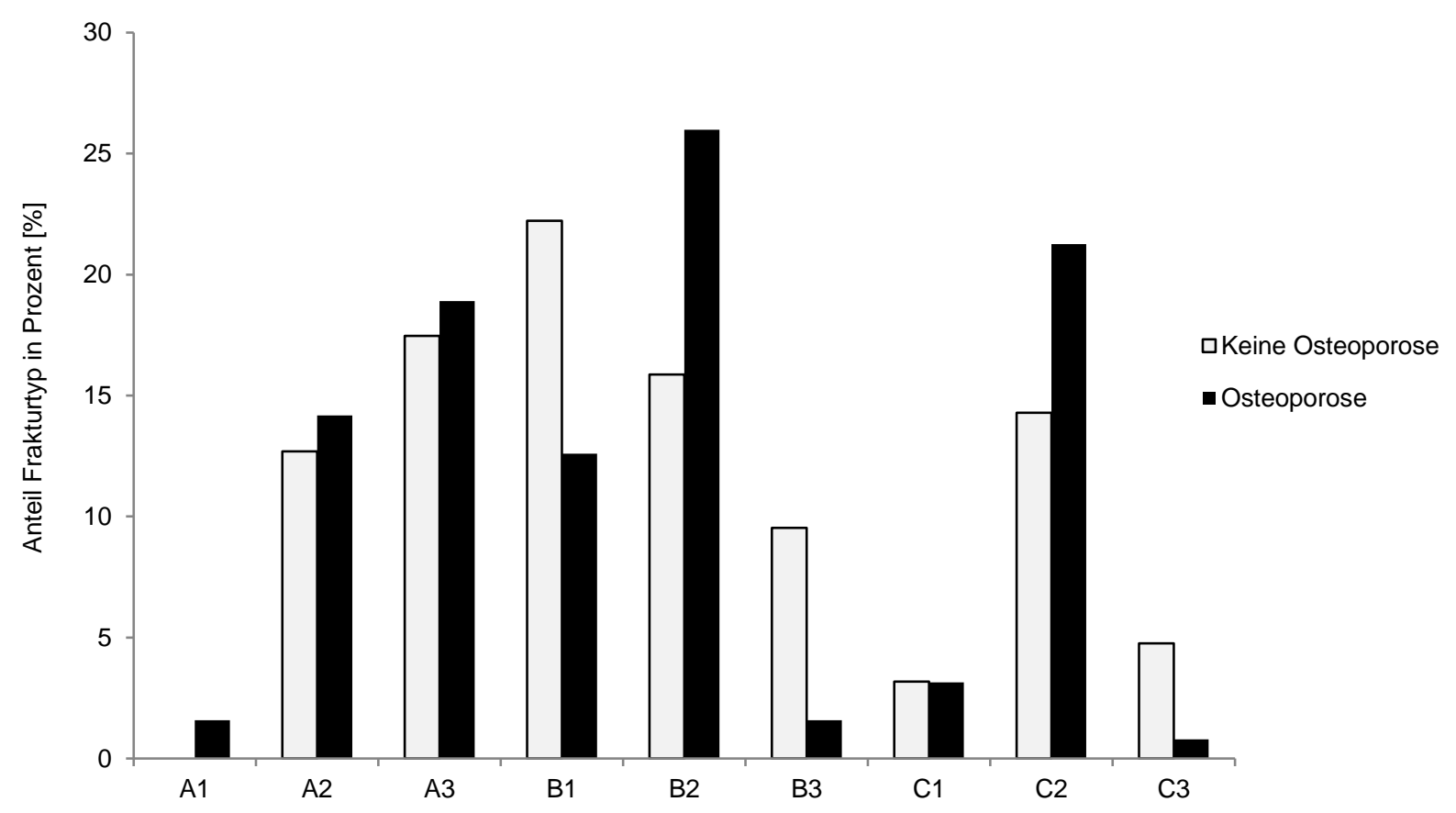

Abb. 12: Frakturverteilung nach AO/OTA-Klassifikation in beiden Gruppen. Angaben in Prozent [\%]. Bei Patienten mit Osteoporose zeigten sich häufiger Frakturen vom Typ B2 und Typ C2. In der Kontrollgruppe waren B1-Frakturen, B3-Frakturen und C3-Frakturen häufiger. Diese Unterscheide waren ohne statistische Signifikanz.

In der Osteoporosegruppe fanden sich damit tendenziell häufiger Frakturen vom Typ B2 $(26,0 \%)$ und stark dislozierte Frakturen vom Typ C2 $(21,3 \%)$ als in der Kontrollgruppe $(15,9 \%$ und $14,3 \%)(p=0,12 ; p=0,25)$. In der Kontrollgruppe hingegen waren Frakturen vom Typ B1 (22,2\%) und Luxationsfrakturen vom Typ B3 (9,5\%) und C3 (4,8\%) häufiger als in der Osteoporosegruppe $(12,6 \%, 1,6 \%$ und $0,8 \%)(p=0,09 ; p=0,06 ; p=0,11)$. Diese Unterschiede waren ohne statistische Signifikanz. A1-Frakturen waren insgesamt selten und traten nur in der Osteoporosegruppe auf (1,6\%). Die Verteilung der A2-, A3- und C1-Frakturen war in beiden Gruppen ähnlich: Osteoporosegruppe 14,2\%, 18,9\%, 3,1\%; Kontrollgruppe 12,7\%, $17,4 \%, 3,1 \% ; p=0,78, p=0,81, p=1.00$. 


\subsection{Primäres Repositionsergebnis}

Eine anatomische Reposition konnte bei 176 Patienten (92,6\%) erreicht werden. Bei Patienten mit Osteoporose entsprach dies 119 (93,7\%) Fällen und bei Patienten ohne Osteoporose 57 Fällen (90,5\%) (siehe Abb. 13). Diesbezüglich gab es zwischen den Gruppen keinen statistisch signifikanten Unterschied $(p=0,42)$.

\section{Anatomische Reposition}

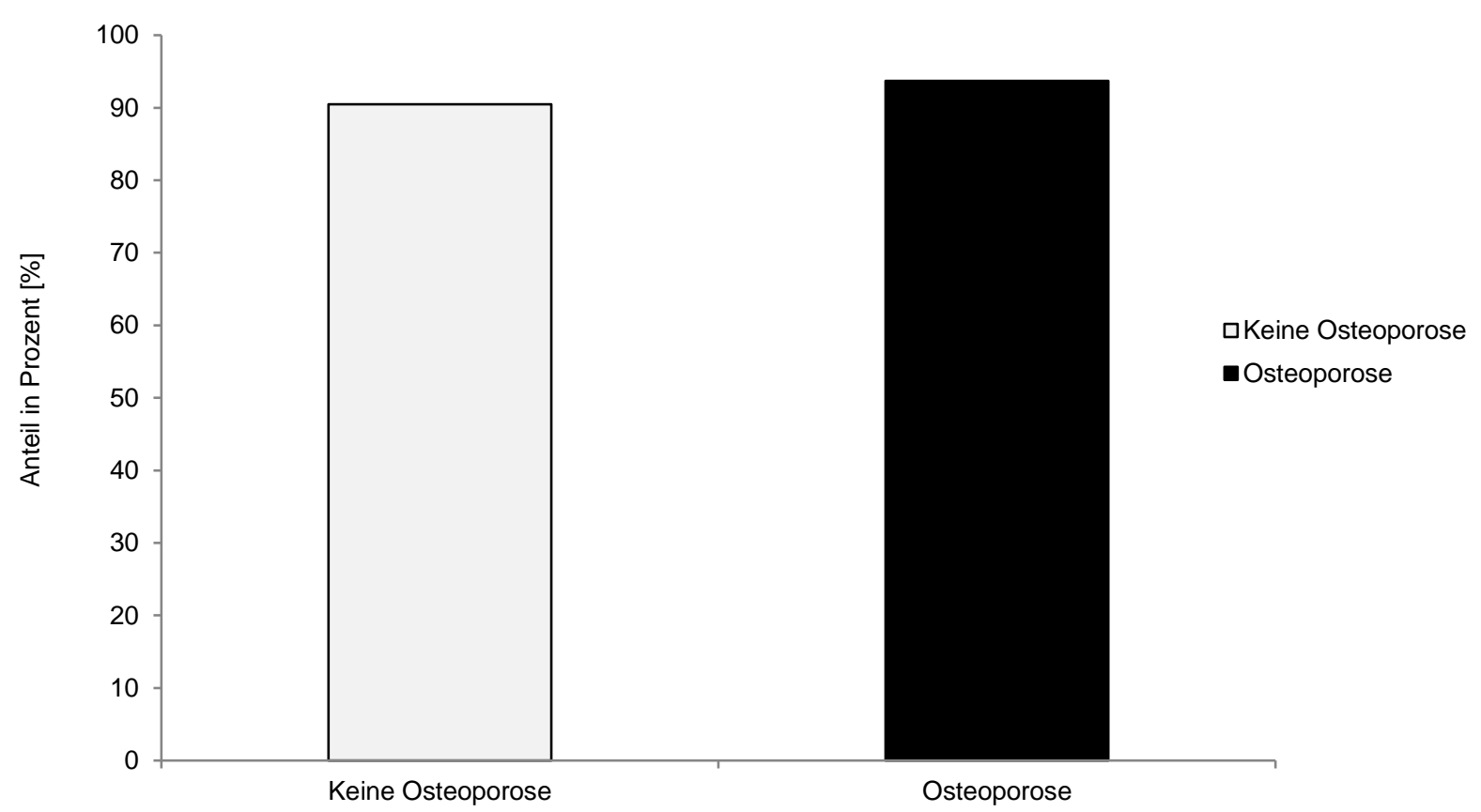

Abb. 13: Anteil der postoperativen anatomischen Reposition. Angaben in Prozent [\%]. In der Osteoporosegruppe konnte in 93,7\%, in der Gruppe ohne Osteoporose in 90,5\% der Fälle eine anatomische Reposition erreicht werden. Es zeigte sich kein statistisch signifikanter Unterschied $(p=0,42)$. 


\subsection{Sekundäre Dislokation}

Bei 37 (19,5\%) aller Patienten ließ sich im Verlauf eine sekundäre Dislokation beobachten, wobei in $83,8 \%$ der Fälle eine Osteoporose vorlag. In der Osteoporosegruppe waren 31 $(24,4 \%)$ und in der Kontrollgruppe 6 (9,5\%) der Patienten betroffen (siehe Abb.14). Dieser Unterschied war statistisch signifikant $(p<0,05)$.

\section{Sekundäre Dislokation}

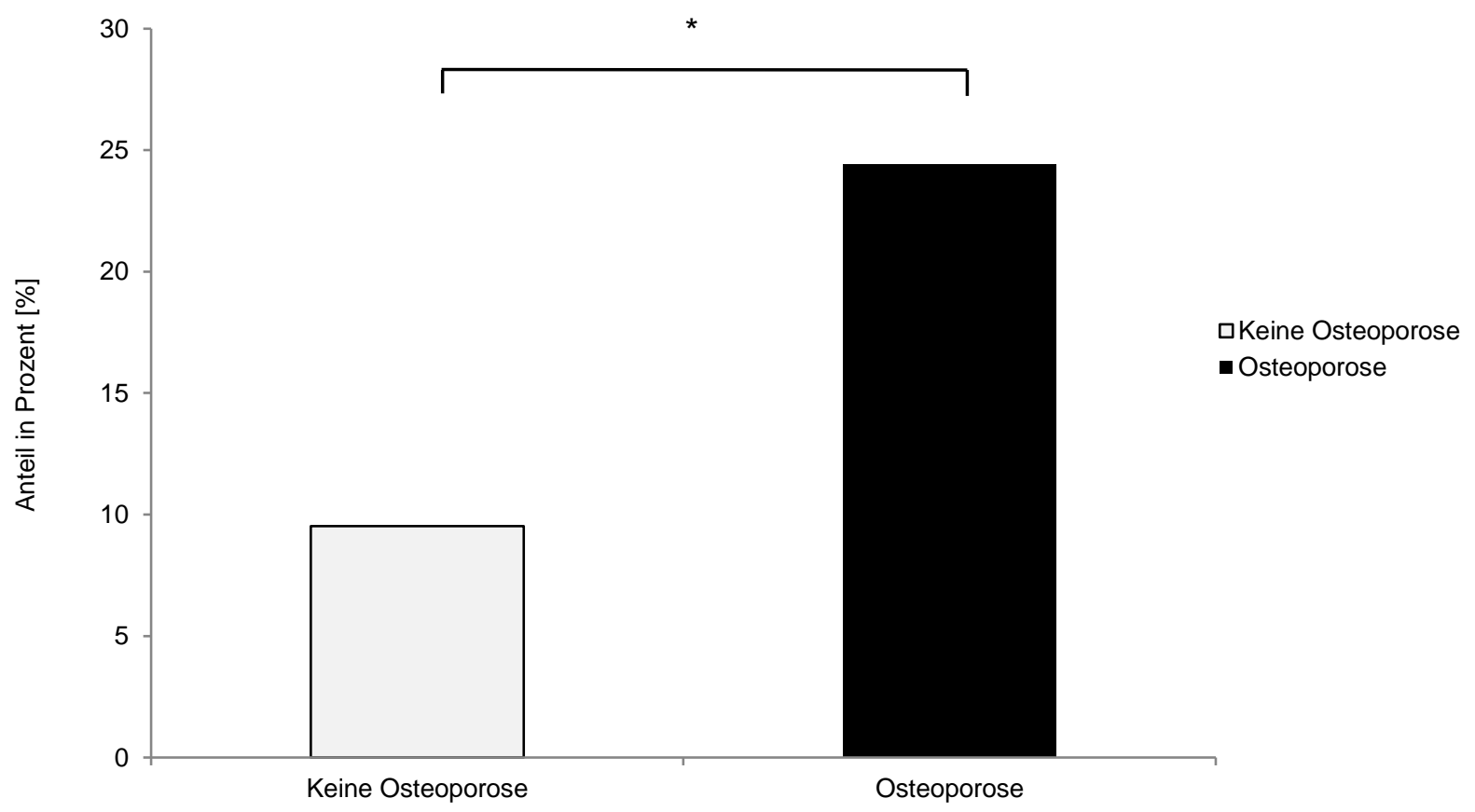

Abb. 14: Anteil sekundärer Dislokationen in der Osteoporosegruppe und Kontrollgruppe. Angaben in Prozent [\%]. In der Osteoporosegruppe war mit $24,4 \%$ der Fälle eine sekundäre Dislokation signifikant häufiger als in der Kontrollgruppe mit 9,5\% $\left({ }^{*} \mathrm{p}<0,05\right)$ 
Bei den 31 Fällen in der Osteoporosegruppe handelte es sich um 10 A3-Frakturen, je 8 B2-, und C2-Frakturen, 2 A2-Frakturen und je eine B1-, B3-, und C3-Fraktur. In der Kontrollgruppe trat bei 2 A3-Frakturen und je einer B2-, B3-, C2-, und C3-Fraktur eine sekundäre Dislokation auf. Somit waren in der Osteoporosegruppe tendenziell sekundäre Dislokationen bei Frakturen vom Typ A3 (41,7\%), B2 (24,2\%) und C2 (29,6\%) häufiger als in der Kontrollgruppe (18,2\%, 10,0\% und 11,1\%) (siehe Abb. 15). Dieser Unterschied war ohne statistische Signifikanz ( $p=0,34, p=0,28, p=0,28)$. Sekundäre Dislokationen bei A2-, und B1Frakturen konnten ausschließlich in der Osteoporosegruppe beobachtet werden. In beiden Gruppen trat keine sekundäre Dislokation bei A1-, und C1-Frakturen auf. Für B3-, und C3Frakturen ergaben sich Dislokationsraten von $50 \%$ bzw. $100 \%$ in der Osteoporosegruppe und $16,7 \%$ bzw. 33,3\% in der Kontrollgruppe. Diese teilweise extremen Werte ergaben sich bei geringen Fallzahlen für B3-, und C3-Frakturen.

\section{Sekundäre Dislokation in Abhängigkeit vom Frakturtyp}

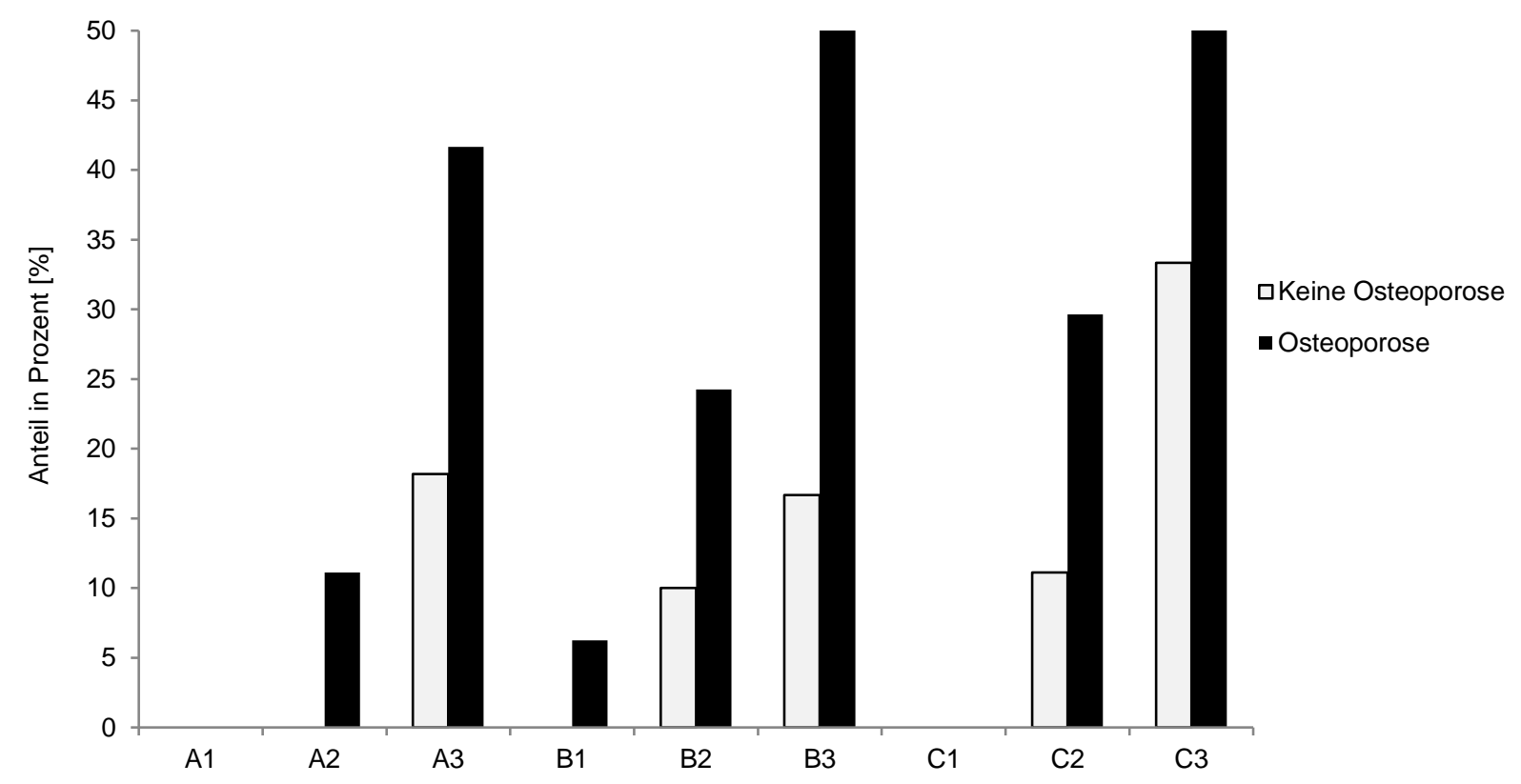

Abb. 15: Anteil sekundärer Dislokationen bezogen auf die Frakturverteilung in der Osteoporosegruppe und der Kontrollgruppe. In der Osteoporosegruppe zeigten sich häufiger Dislokationen bei A3, B2 und C2 Frakturen, jeweils ohne statistische Signifikanz. Extreme Werte ergaben sich für B3-, und C3-Frakturen bei geringen Fallzahlen. 
Unter den impaktierten Frakturtypen (A2, B1, C1) trat in der Osteoporosegruppe in $3(7,9 \%)$ Fällen eine sekundäre Dislokation auf. In der Kontrollgruppe trat keine sekundäre Dislokation auf. Nicht impaktierte Frakturtypen (A3, B2, C2, B3, C3) zeigten in der Osteoporosegruppe in 28 Fällen (32,2\%) und in der Kontrollgruppe in 6 Fällen (15,4\%) eine sekundäre Dislokation (siehe Abb. 16). Dieser Unterschied war statistisch signifikant $(p<0,05)$.

\section{Sekundäre Dislokation impaktierter/nicht impaktierter Frakturen}

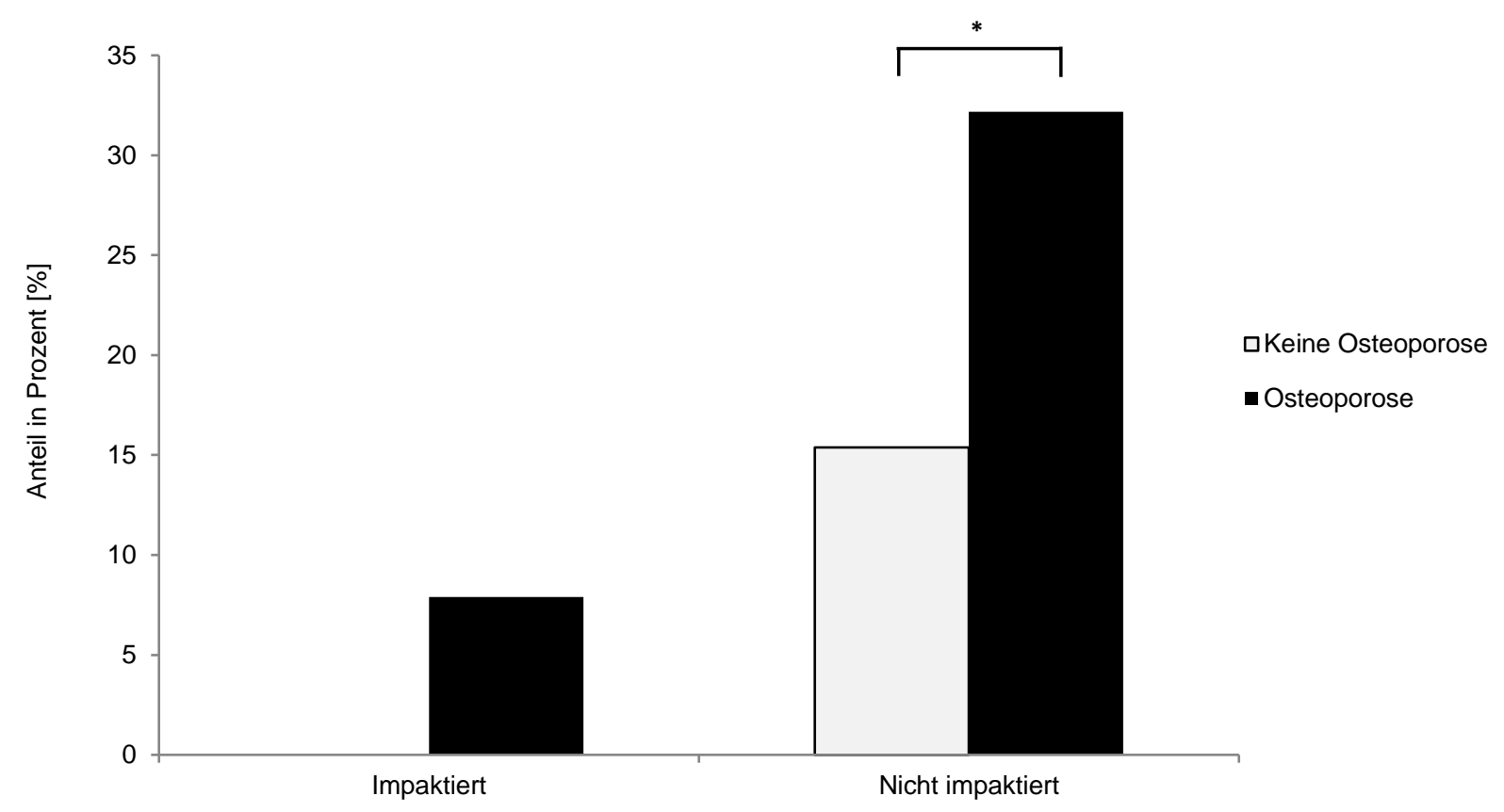

Abb. 16: Anteil sekundärer Dislokationen bei impaktierten und nicht impaktierten Frakturen in der Osteoporose- und der Kontrollgruppe. Nicht impaktierte Frakturtypen dislozierten in der Osteoporosegruppe signifikant häufiger als in der Kontrollgruppe $\left({ }^{*} p<0,05\right)$. Bei impaktierten Frakturen wurden nur in der Osteoporosegruppe sekundäre Dislokationen beobachtet. 


\subsection{Revisionsoperationen}

Revisionsoperationen waren insgesamt bei 27 Patienten (14,2\%) nötig. In der Osteoporosegruppe wurden bei 24 Patienten (18,9\%) Revisionsoperationen durchgeführt. In der Kontrollgruppe war die Anzahl der Revisionsoperationen mit 3 (4,8\%) deutlich geringer (siehe Abb. 17). Hier zeigte sich ein signifikanter Unterschied zwischen den zwei Gruppen $(p<0,01)$.

\section{Revisionsrate}

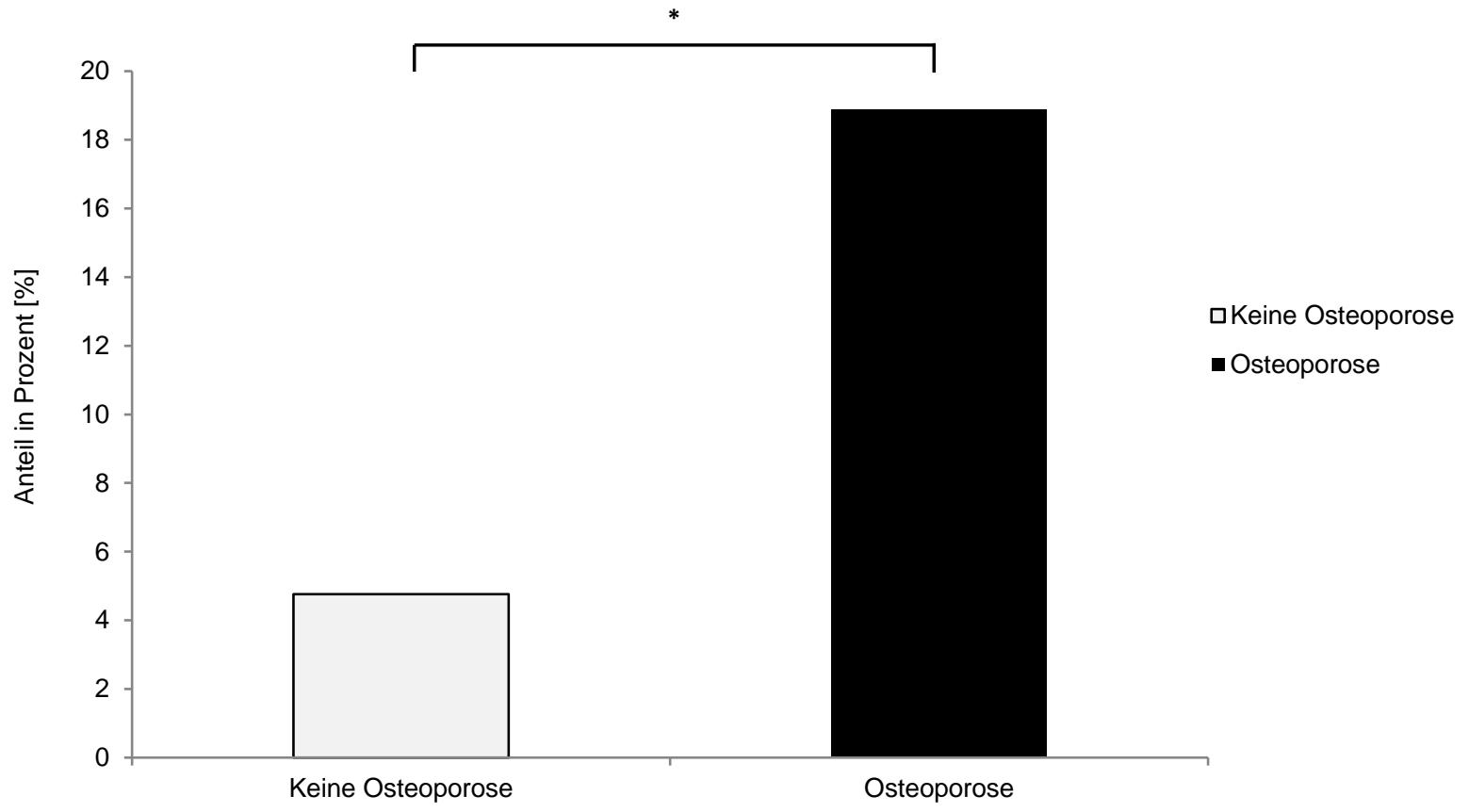

Abb. 17: Notwendige Revisionsoperationen bezogen auf die Osteoporose-, und Kontrollgruppe. In der Osteoporosegruppe waren mit 18,9\% deutlich häufiger Revisionsoperationen nötig als in der Kontrollgruppe (4,8\%). Dieser Unterschied war statistisch signifikant $\left({ }^{*} \mathrm{p}<0,01\right)$. 
In der Osteoporosegruppe entsprach dies 9 A3-Frakturen (37,5\%), 7 B2-Frakturen (21,2\%) und 5 C2-Frakturen (18,5\%), sowie je eine A2-, B1-, und B3-Fraktur. Keine Revision war nötig für A1-, C1-, und C3-Frakturen. In der Kontrollgruppe war lediglich je eine A3-, B2-, und B3-Fraktur revisionspflichtig (siehe Abb. 18). Aufgrund der teilweise geringen Fallzahlen wurde die Auswertung deskriptiv gehalten und nicht statistisch ausgewertet.

\section{Revisionsrate bezogen auf Frakturtyp}

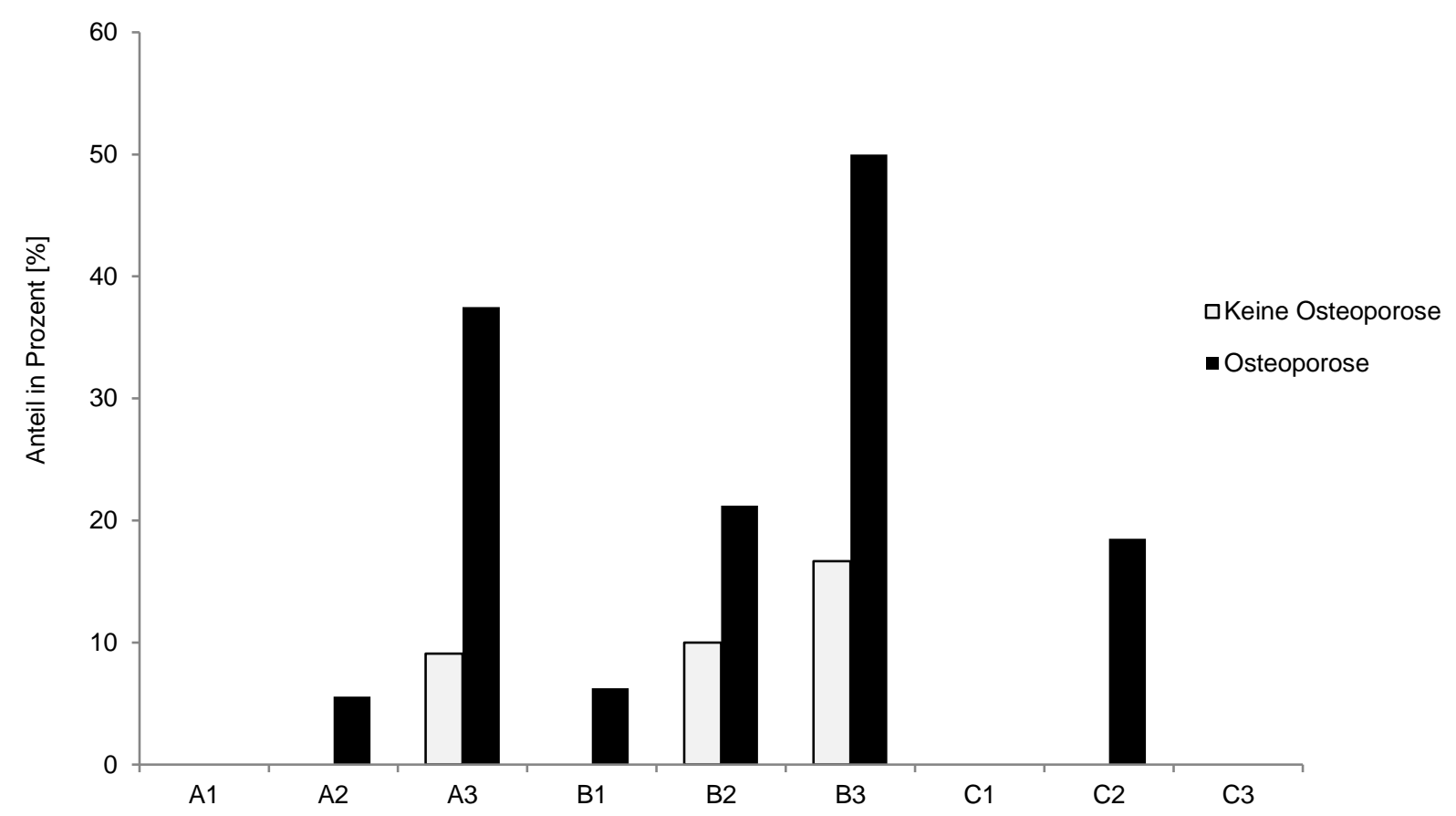

Abb. 18: Revisionsrate bezogen auf den Frakturtyp. Angaben in Prozent. In der Osteoporosegruppe lag die Revisionsrate für A3-Frakturen bei 37,5\%, für B2-Frakturen bei $21,2 \%$ und für C2-Frakturen bei 18,5\%. Bei je einer Revision für A2-, B1-, und B3-Frakturen in der Osteoporosegruppe, sowie in der Kontrollgruppe für A3-, B2-, und B3-Frakturen, war bei geringer Fallzahl die statistische Aussagekraft eingeschränkt. 
Für impaktierte Frakturtypen wurden in der Osteoporosegruppe 2 (5,3\%) und in der Kontrollgruppe keine Revisionsoperationen registriert. Nicht impaktierte Frakturtypen mussten in der Osteoporosegruppe mit 22 Fällen (25,3\%) signifikant häufiger revidiert werden als in der Kontrollgruppe mit 3 Fällen $(7,7 \% ; p<0,05)$ (siehe Abb. 19).

\section{Revisionsrate impaktierter/nicht impaktierter Frakturen}

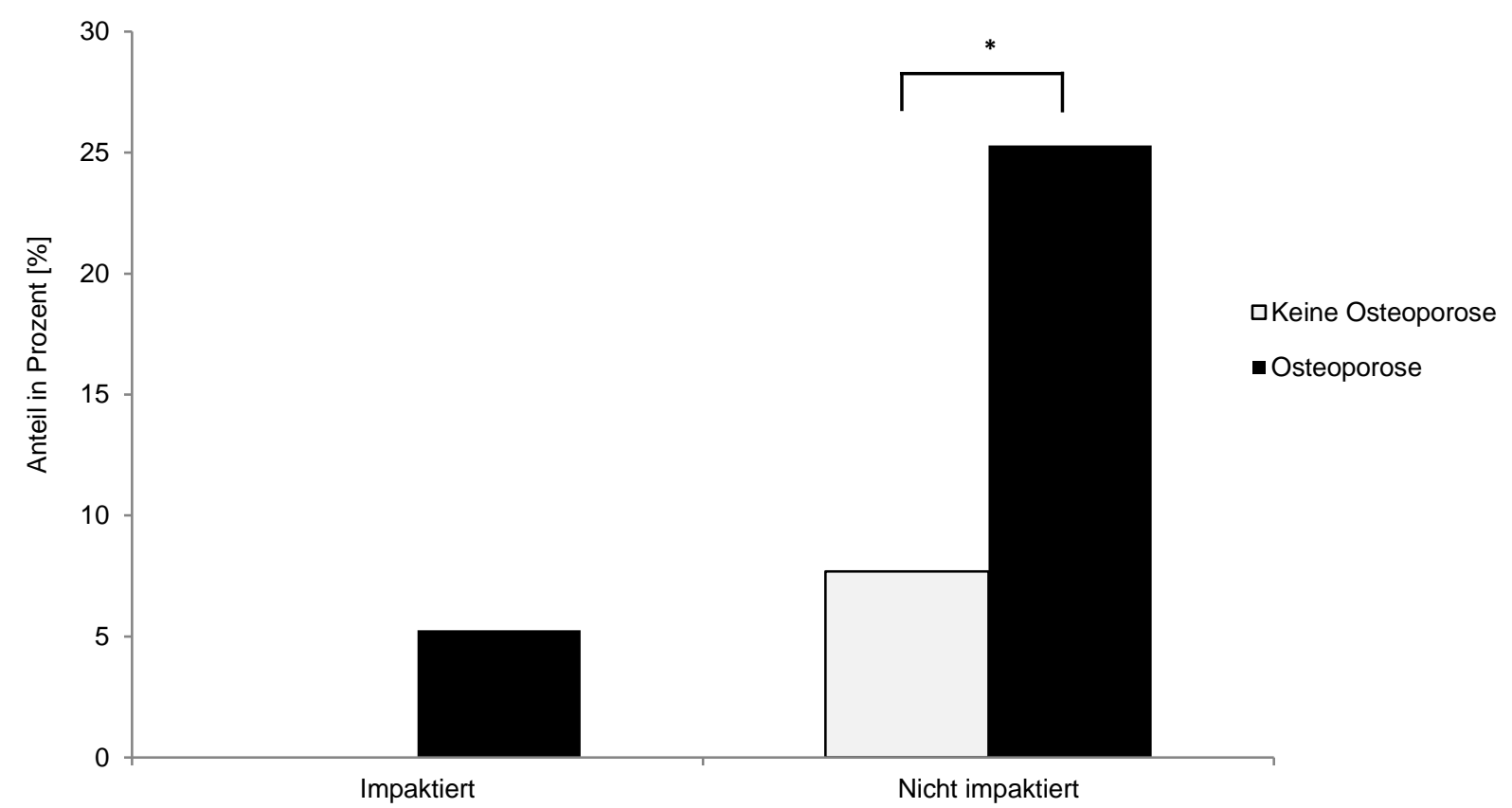

Abb. 19: Anteil der Revisionen bei impaktierten und nicht impaktierten Frakturen in der Osteoporose- und der Kontrollgruppe. Nicht impaktierte Frakturtypen mussten in der Osteoporosegruppe signifikant häufiger revidiert werden als in der Kontrollgruppe $\left({ }^{*} p<0,05\right)$. Bei impaktierten Frakturen wurden nur in der Osteoporosegruppe Revisionsoperationen beobachtet. 
Abbildung 20 gibt einen Überblick über die Art der Revisionsoperationen in beiden Gruppen. Insgesamt wurden 13 Re-Osteosynthesen (48,1\%) und 8 Metallentfernungen (29,6\%) durchgeführt. Der Wechsel auf eine Endoprothese war in 4 (14,8\%) bzw. zu einer inversen Schulterprothese in 2 (7,4\%) Fällen nötig.

In der Osteoporosegruppe wurden 11 Re-Osteosynthesen (45,8\%) und 7 Metallentfernungen $(29,3 \%)$ durchgeführt, sowie 4 Hemiprothesen (16,7\%) und 2 inverse Schulterprothesen $(8,3 \%)$ implantiert. In der Kontrollgruppe war eine Metallentfernung (33,3\%) und 2 ReOsteosynthesen $(66,7 \%)$ nötig.

\section{Art der Revisionsoperationen}

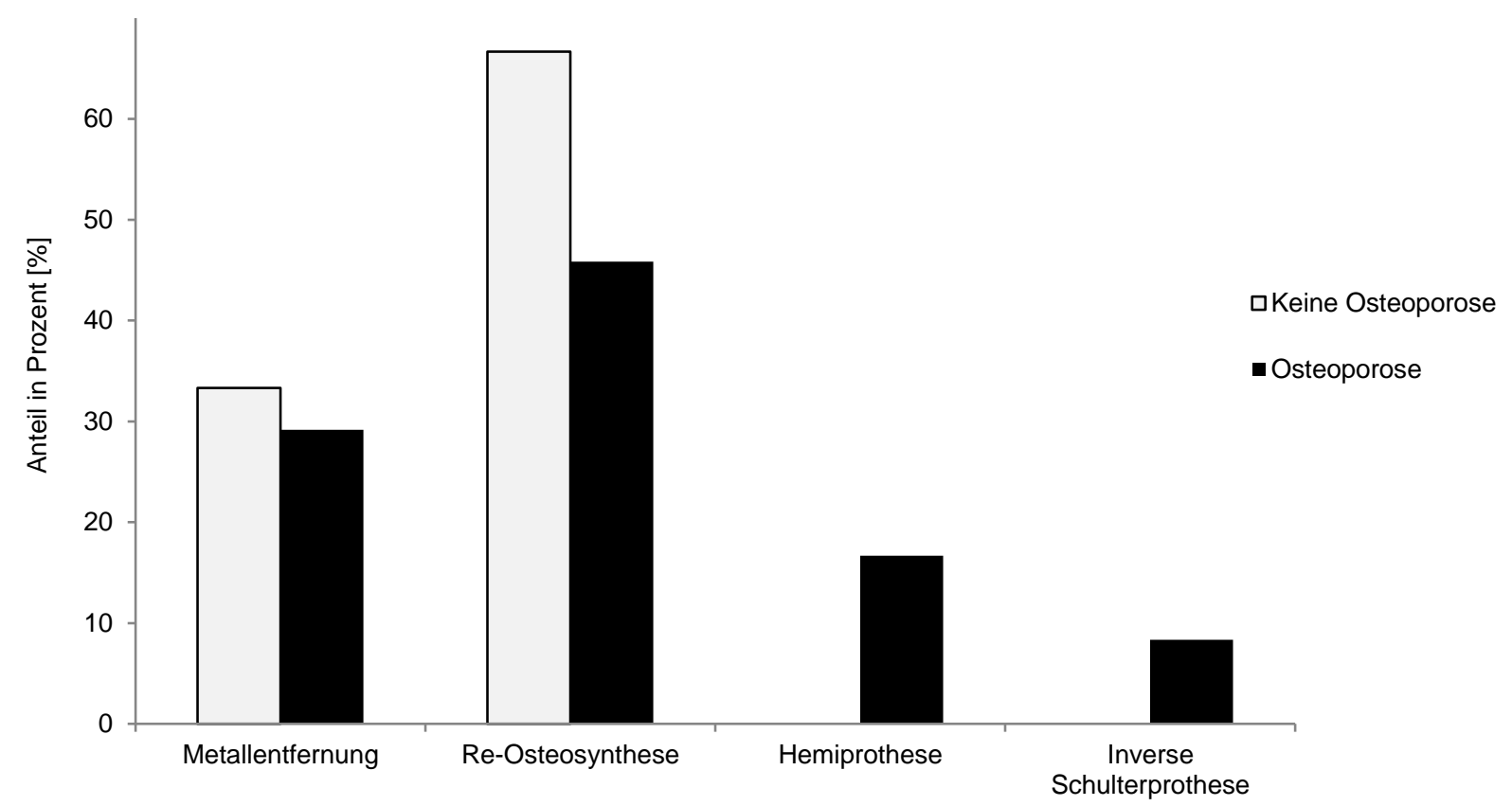

Abb. 20: Überblick über die Art der durchgeführten Revisionsoperationen. Die Re-Osteosynthese war das am häufigsten gewählte Revisionsverfahren in beiden Gruppen (48,1\%). Nur bei Patienten mit Osteoporose war der Wechsel auf eine Hemiprothese (16,7\%) bzw. inverse Schulterprothese $(8,3 \%)$ nötig. 


\section{Diskussion}

Ziel der vorliegenden Studie war es, den Einfluss der Osteoporose auf den Frakturtyp, die Rate der sekundären Dislokation nach winkelstabiler Plattenosteosynthese und Rate sowie Art der nötigen Revisionsoperationen nach winkelstabiler Plattenosteosynthese bei der proximalen Humerusfraktur zu untersuchen. Im Folgenden werden die Methodik der Studie, die Ergebnisse sowie Limitationen der vorliegenden Arbeit diskutiert.

\subsection{Diskussion der Methoden}

Die vorliegende Studie entspricht einer retrospektiven Observationsstudie. Das minimale Follow-up lag bei 24 Monaten und die Follow-up Rate betrug 89,2\%. Diese Parameter entsprechen den Standardanforderungen der höherklassigen, international anerkannten wissenschaftlichen Journale [43].

Die Knochendichtemessung wurde mittels DXA von Lendenwirbelsäule und proximalem Femur durchgeführt. Dies entspricht dem Goldstandard nach den Empfehlungen der WHO [1]. In vergleichbaren Studien zur Knochendichte am proximalen Humerus findet man eine starke Heterogenität in der Definition von Osteoporose und der Methoden zur Bestimmung der Knochendichte, was Goldhahn et al. als Ursache für die schlechte Vergleichbarkeit der Ergebnisse angibt [55]. In den 10 untersuchten Studien dieser Übersichtsarbeit wurde sechs mal der Singh-Index, zwei mal DXA, einmal der Barnett-Nodin-Index und einmal die ICD-9CM Diagnose benutzt [55]. Krappinger et al. berechnete in einer Studie die Knochendichte des kontralateralen Humerus mit Hilfe einer Computertomografie und versucht so auf die betroffene Seite zu schließen [39]. Hepp et al. wiederum verwendete den Cortical Index als Parameter für die Knochendichte [56]. In zukünftigen Studien sollte die Bestimmung der Knochendichte wie in der vorliegenden Studie gemäß Empfehlung der WHO mittels DXA erfolgen, damit bessere Vergleichbarkeit zwischen verschiedenen Studien geschaffen wird. Außerdem ist zu beachten, dass die DXA Messung während des stationären Aufenthaltes, also in unmittelbarem zeitlichen Zusammenhang zum Frakturereignis stattgefunden hat. Hier handelt es sich um ein alleinstellungsmerkmal und um einen methodischen Vorteil gegenüber Studien, die aus Krankenkassendatenbanken Diagnosecodes korrelieren.

Die Fraktureinteilung erfolgte gemäß der AO/OTA-Klassifikation anhand von Röntgenaufnahmen in True-a.p. und y-view Technik. In der Literatur hat sich die AO/OTA Klassifikation und die Neer Klassifikation etabliert [25]. Die AO/OTA-Klassifikation wurde in 
der Vergangenheit auf Grund ihrer schlechten Reproduzierbarkeit und Interobserverreliabilität kritisiert [57]. In dieser Studie wurde dennoch die AO/OTAKlassifikation gewählt, weil sie eine sehr genaue Einteilung der Frakturen möglich macht, insbesondere in Bezug auf die Varus und Valgusdislokation bzw. auf die Impaktation der Fraktur. Auch Court-Brown et al. favorisierte in einer großen epidemiologischen Studie zur Frakturmorphologie die AO/OTA-Klassifikation aus diesem Grund [28]. Um die Reproduzierbarkeit zu verbessern, wurde bei erschwerter Beurteilbarkeit eine Computertomografie angefertigt. Hirzinger et al. empfiehlt den routinemäßigen Einsatz von computertomografischen 3-D-Rekonstruktionen, um eine noch bessere Beurteilung der Frakturmorphologie zu ermöglichen [61]. Diese Methode wurde in der vorliegenden Studie nicht standardmäßig verwendet und nur bei Bedarf angefordert. Eine differenzierte Betrachtung der impaktierten (A2, B1, C1) und nicht impaktierten Frakturen (A3, B2, C2, B3, C3), die in der von von Resch et al. publizierte Frakturklassifikation beschrieben wurden, schien sinnvoll, da der Grad der Impaktation einen starken Einfluss auf die Prognose hat [26]. So berichteten Solberg et al. von einem deutlich schlechteren funktionellen Ergebnis bei Patienten mit nicht impaktierten Frakturen und kritisiert die mangelnde Beschreibung dieser Frakturen in der AO/OTA Klassifikation [62]. Eine solche differenzierte Betrachtung der Frakturen wurde bereits in der Literatur verwendet [63].

Zur operativen Versorgung proximaler Humerusfrakturen haben sich zahlreiche Verfahren etabliert [27, 32, 37-40]. Da in dieser Studie ausschließlich die winkelstabile Plattenosteosynthese als Osteosyntheseverfahren untersucht wurde, ist kein Vergleich zwischen verschiedenen Therapieverfahren möglich. Die Indikationsstellung zur Operation gemäß Neer-Kriterien, entspricht einem etabliertem Standard [42, 53]. Als operativer Zugang wurde der deltoideopektorale Zugang gewählt. Hepp et al. konnten hierfür im Vergleich zum Delta-Splitting-Zugang bessere funktionelle Langzeitergebnisse erzielen [64]. Das postoperative Procedere war mit dem Vorgehen in anderen Studien vergleichbar [43, 53]. Nicht Gegenstand der Untersuchung waren der Zeitpunkt der Operation nach Trauma, die Operationsdauer und die Dauer des stationären Aufenthalts. Inwiefern diese Parameter durch Osteoporose beeinflusst wurden, konnte daher nicht untersucht werden und sollte in nachfolgende Studien einbezogen werden, insbesondere im Hinblick auf gesundheitsökonomische Fragestellungen. 


\subsection{Diskussion der Ergebnisse}

\subsubsection{Patienten}

Das Durchschnittsalter aller Patienten war mit 74,3 $( \pm 9,9)$ etwas höher als in vergleichbaren Studien: Sudkamp et al. gaben 62,9 ( $\pm 15,7)$ [42] und Krappinger et al. 63,9 $( \pm 12,6)$ Jahre an [39]. Der Wert ist aber unter Anbetracht der epidemiologischen Studie von Kristiansen et al. nicht überraschend [8]. In der vorliegenden Studie zeigte sich bei Patienten mit Osteoporose ein signifikant höheres Durchschnittsalter $(75,7 \pm 9,3)$ als bei Patienten ohne Osteoporose $(71,3 \pm 10,3)$. Dieser Unterschied der beiden Gruppen kann durch die Korrelation von steigendem Alter und zunehmender Osteoporoseprävalenz erklärt werden. Der höhere Anteil der Frauen (86,3\%) als der der Männer (13,7\%) kann als eine Konsequenz aus der mit dem Alter stark ansteigenden Inzidenz proximaler Humerusfrakturen bei Frauen angesehen werden [8]. Sudkamp et al. und Krappinger et al. beschreiben den Anteil der Frauen mit $72,0 \%$ bzw. $71,1 \%[39,42]$.

Im Gesamtkollektiv lag die Osteoporoseprävalenz bei 66,8\%. Die Prävalenz lag damit deutlich höher als für die Normalbevölkerung in Deutschland angegeben (14\% gesamt; 24\% Frauen, 6\% Männer) [4]. In der vorliegenden Studie wurden lediglich Patienten mit operativ versorgter proximaler Humerusfraktur eingeschlossen, wodurch es sich um ein selektioniertes Patientenkollektiv handelt. Untersuchungen zur Prävalenz von Osteoporose in einem allgemeinen unfallchirurgischen Patientenkollektiv zeigten, dass bei $56,6 \%$ der Patienten mit Fraktur eine Osteoporose mit DXA nachgewiesen werden konnte [2]. Roux et al. dagegen konnten unter konservativ und operativ behandelten Patienten lediglich eine Osteoporoseprävalenz von 34\% nachweisen [20]. Es bleibt zu klären, ob Osteoporose das Risiko für einen komplizierteren Bruch erhöht, der in der Folge einer Operation bedarf und dadurch die höhere Prävalenz im operativ behandelten Kollektiv bedingt. Dies könnte Gegenstand nachfolgender Untersuchungen sein, die Patienten mit konservativ therapierter proximaler Humerusfraktur ebenfalls einer Knochendichtemessung mittels DXA unterziehen.

Hinsichtlich der Osteoporoseprävalenz zwischen Frauen (67,7\%) und Männern (61,5\%) konnte kein statistisch signifikanter Unterschied beobachtet werden. Dies steht im Widerspruch mit den Daten der Osteoporoseprävalenz für Deutschland im Jahr 2009 (Frauen 24\%, Männer 6\%) [4]. Auch Haasters et al. konnten keinen Unterschied der geschlechtsspezifischen Osteoporoseprävalenz nachweisen (Frauen 56,2\%, Männer 59\%, eingeschlossen wurden Patienten mit Frakturen der BWS und LWS, des proximalen Femur, proximalen Humerus und distalen Radius) [2]. In derselben Studie konnte gezeigt werden, dass Männer signifikant seltener eine Knochendichtemessung mittels DXA erhalten [2]. Vor 
diesem Hintergrund zeigt sich die Wichtigkeit, auch bei Männern auf eine angemessene Osteoporoseabklärung zu achten, insbesondere wenn bereits eine Fraktur vorliegt. 


\subsubsection{Anatomische Reposition}

Die Rate der operativ erreichten anatomischen Repositionen war in beiden Gruppen vergleichbar (Osteoporosegruppe: 93,7\%, Kontrollgruppe: 90,5\%). Hardeman et al. konnten in einer retrospektiven Studie mit 307 Frakturen zeigen, dass eine anatomische Reposition mit weniger Komplikationen und Revisionseingriffen korreliert. Beim Vorliegen einer medialen Stufenbildung über $2 \mathrm{~mm}$ zeigte sich eine höhere Komplikationsrate (20\%) und Revisionsrate (27,8\%) als bei einer Stufenbildung unter $2 \mathrm{~mm} \mathrm{(6,8 \%} \mathrm{und} \mathrm{16,7 \% )} \mathrm{[49].} \mathrm{In} \mathrm{der}$ vorliegenden Studie wurde eine anatomische Reposition mit Stufenbildung unter $2 \mathrm{~mm}$ und Achsabweichung unter $10^{\circ}$ definiert und erfüllt damit auch die Kriterien von Hardeman. Durch die annähernd gleiche Verteilung der anatomischen Reposition bei Patienten mit und ohne Osteoporose, war diesbezüglich das Risiko für eine nachfolgende sekundäre Dislokation oder Revisionsoperation in beiden Gruppen vergleichbar.

\subsubsection{Frakturverteilung}

Court-Brown et al. schlussfolgerten aus der mit dem Alter zunehmenden Inzidenz komplexerer Frakturformen einen Zusammenhang mit Osteoporose [28]. Im Rahmen der vorliegenden Studie konnte kein statistisch signifikanter Effekt der Osteoporose auf die Frakturmorphologie gefunden werden. Obwohl bei Patienten mit Osteoporose häufiger varisch dislozierte B2-Frakturen und C2-Frakturen auftraten, als dies bei Patienten ohne Osteoporose der Fall war, zeigte sich keine statistische Signifikanz. Die fehlende statistische Aussagekraft diesbezüglich könnte mitbegründet sein in der teilweise geringen Fallzahl der einzelnen Frakturtypen. Weitere Studien mit ausreichender Fallzahl, die den Einfluss einer mit DXA-Messung diagnostizierten Osteoporose auf den Frakturtyp nach AO/OTAKlassifikation adressieren, sind nötig um diesen Zusammenhang abschließend zu klären. 


\subsubsection{Sekundäre Dislokation}

In der Literatur findet man für die winkelstabile Plattenosteosynthese Komplikationsraten von 9 - 48,8\% [42, 43, 47, 48, 65], wobei die Begriffsdefinition der Komplikation nicht einheitlich ist. Während Thanasas et al. in einer Übersichtsarbeit eine Rate sekundärer Dislokationen von 12,2\% beschrieben [43], wird diese von Sproul et al. gar nicht erst definiert und durch varische Fehlheilung und Schraubenperforation umschrieben [47]. Solberg et al. beschrieben eine Rate sekundärer Dislokationen von $13 \%$ für varische bzw. $7 \%$ für valgische Frakturmuster [38]. In einer Studie von Lee et al. beträgt die Rate sekundärer Dislokationen 11,4\% [66]. Der Einfluss einer vorliegenden Osteoporose auf die Rate sekundärer Dislokationen wurde in keiner der genannten Studien näher untersucht. In der vorliegenden Studie lag die Rate sekundärer Dislokationen im Gesamtkollektiv bei 19,5\%. Dieser Wert liegt etwas höher als in oben genannten Studien. Unter dem Begriff der sekundären Dislokation wurden postoperativ auftretende Fragmentdislokation über $2 \mathrm{~mm}$, Achsabweichung über $10^{\circ}$, Perforation (Cut-Out) der Schrauben in das Glenohumeralgelenk und Dislokation oder Bruch des Osteosynthesematerials zusammengefasst. Eine gemeinsame Betrachtung von Schraubenperforation und Fragmentdislokation bzw. Achsabweichung wurde zum einen wegen des häufigen simultanen Auftretens [48], zum anderen wegen der erleichterten Auswertung gewählt. Auf diese Weise wurden relevante Komplikationen erfasst, die häufig zu Revisionsoperationen führen und sich auf die Interaktion zwischen Knochen und Osteosynthesematerial beziehen [43, 53]. Andere Komplikationen, wie die avaskuläre Humeruskopfnekrose, subacromiales Impingement und Infektionen wurden nicht untersucht.

Bei Patienten mit Osteoporose trat eine sekundäre Dislokation mit 24,4\% signifikant häufiger auf als bei Patienten ohne Osteoporose (9,5\%). Bislang wurde der Einfluss von Osteoporose auf die winkelstabile Plattenosteosynthese nicht ausreichend untersucht [55]. Dass eine niedrige Knochendichte einen negativen Einfluss auf die stabile Verankerung des Osteosynthesematerials im Knochen hat, konnte biomechanisch gezeigt werden [54]. In klinischen Studien konnte dieser Zusammenhang allerdings bislang nicht abschließend geklärt werden [55]. Krappinger et al. verwendeten CT-Daten des kontralateralen Humerus für die Knochendichtemessung. In dieser prospektiven Studie mit 67 Patienten korrelierten niedrigere Werte der kontralateralen Seite, mit einem erhöhten Risiko für Komplikationen [39]. Im Gegensatz dazu konnten Hepp et al. In einer Matched-Pair Analyse mit 113 Patienten keinen Zusammenhang zwischen Knochendichte und Komplikationsrate beobachten [56]. Als Parameter für die Knochendichte wurde der Cortical Index verwendet. Jung et al. wiederum beschrieben Osteoporose als unabhängigen Risikofaktor für eine sekundäre Dislokation [67], verwendeten allerdings einen retrospektiven Ansatz mit 
multivariater Regression bei kleiner Gruppengröße und empfiehlt die Durchführung prospektiver Studien. Die Ergebnisse der vorliegenden Studie zeigen eine signifikante Erhöhung des Risikos für Komplikationen nach winkelstabiler Plattenosteosynthese bei Patienten mit Osteoporose. Dies spricht für eine Verschlechterung der Stabilität des Osteosynthesematerials im osteoporotischen Knochen nach winkelstabiler Plattenosteosynthese.

\subsubsection{Revisionsrate}

Eine Revisionsoperation war im Gesamtkollektiv in $14,9 \%$ der Fälle (27 Revisionsoperationen) nötig und ist mit Werten aus der Literatur vergleichbar. Sproul et al. beschrieben in einer Analyse von 12 Studien mit insgesamt 514 Patienten eine Revisionsrate von 13,8\% (71 Revisionsoperationen) [47]. Thanasas et al. gaben in einer ähnlichen Übersichtsarbeit über 12 Studien mit insgesamt 791 Patienten eine Revisionsrate von 13,7\% (92 Revisionsoperationen) an [43]. In einer retrospektiven, klinischen Studie mit 53 Patienten konnten von Owsley et al. eine Revisionsrate von 13\% (7 Revisionsoperationen) gefunden werden [48]. Überblickend betrachtet, zeigt sich nur eine geringe Streuung der Revisionsrate in den genannten Studien. Ob Osteoporose das Risiko für Revisionsraten erhöht, wurde in keiner der genannten Studien untersucht.

Betrachtet man die Gruppen separat, zeigte sich in der Osteoporosegruppe dieser Studie eine signifikant höhere Revisionsrate von $18,9 \%$ als bei Patienten ohne Osteoporose $(4,8 \%)$. Wie oben beschrieben, war in der Osteoporosegruppe die Rate der sekundären Dislokation signifikant erhöht. In Folge dessen ist auch eine erhöhte Revisionsrate beim Vorliegen einer Osteoporose naheliegend. Demnach kann Osteoporose auch als Risikofaktor für Revisionsoperationen nach winkelstabiler Plattenosteosynthese am proximalen Humerus diskutiert werden.

\subsubsection{Sekundäre Dislokation und Revision bezogen auf den Frakturtyp}

Die positive Korrelation der Schwere der Fraktur mit dem Risiko für nachfolgende sekundäre Dislokation wurde mehrfach beschrieben: In einer prospektiven Multicenterstudie mit 131 Patienten konnten Röderer et al. den Frakturtyp nach AO/OTA-Klassifikation als stärksten Risikofaktor für das Auftreten einer sekundären Dislokation identifizieren. 80\% der Patienten mit sekundärer Dislokation mussten sich einer Revisionsoperation unterziehen [68]. Der 
Einfluss einer Osteoporose als Begleiterkrankung wurde in diesem Zusammenhang nicht untersucht. Owsley et al. konnten keinen Zusammenhang zwischen Frakturtyp und Komplikationsrate nachvollziehen. Allerdings zeigten sich für Patienten über 60 Jahren mit Schraubenperforation unterschiedliche Revisionsraten für 3-, und 4-Fragmentfrakturen (75\%) und 2-Fragmentfrakturen (0\%) [48]. Osteoporose wurde hier wiederum nicht als Risikofaktor untersucht, allerdings kann das hohe Alter möglicherweise einen Hinweis auf eine erhöhte Osteoporoseprävalenz darstellen.

In der vorliegenden Studie konnte der Einfluss der Frakturmorphologie auf die Rate der sekundären Dislokation und Revision analysiert werden. Betrachtet man die Rate der sekundären Dislokationen bezogen auf die Frakturverteilung gemäß AO/OTA in beiden Gruppen, zeigte sich kein statistisch signifikanter Unterschied zwischen den Gruppen, auch wenn in der Osteoporosegruppe A3-Frakturen, B2-Frakturen und C2 Frakturen tendenziell häufiger sekundär dislozierten, als bei Patienten ohne Osteoporose. Die kumulative Betrachtung impaktierter Frakturtypen (A2, B1, C1) und nicht impaktierter Frakturtypen (A3, B2, C2, B3, C3) zeigte jedoch signifikante Unterschiede zwischen den Gruppen. Während es unter den impaktierten Frakturen keinen Unterschied zwischen den Gruppen gab, war für nicht impaktierte Frakturen die Rate sekundärer Dislokationen und der Revision in der Osteoporosegruppe signifikant höher als in der Kontrollgruppe. Nach den Ergebnissen der vorliegenden Studie haben nicht impaktierte Frakturtypen bei gleichzeitig bestehender Osteoporose ein stark erhöhtes Risiko einer sekundären Dislokation und nachfolgenden Revisionsoperationen, was auch mit dem von Solberg et al. beschriebenen schlechteren Outcome für nicht impaktierte Frakturen vereinbar ist [62]. So scheint insbesondere der Grad der Impaktation und Varus-, bzw. Valgusfehlstellung ein Risikofaktor für nachfolgende Komplikationen zu sein [62].

Fasst man die Ergebnisse der vorliegenden Studie zusammen zeigt sich bei Patienten mit Osteoporose, die eine nicht impaktierte Fraktur erleiden, ein signifikant erhöhtes Risiko für nachfolgende sekundäre Dislokation und Revisionsoperation nach winkelstabiler Plattenosteosynthese. Vor diesem Hintergrund müssen alternative primäre Therapieverfahren diskutiert werden, die mit weniger Komplikationen und Revisionsoperationen behaftet sind. Betrachtet man die Ergebnisse der Studie, findet sich für A3-Frakturen eine sekundäre Dislokation in $41,7 \%$ der Fälle. Die A3-Fraktur ist definitionsgemäß auf Grund der fehlenden Impaktation und der Dislokation instabil und bedarf oftmals einer operativen Versorgung. Dies wurde auch schon vor Einführung der AO/OTA-Klassifikation von Neer für die dislozierte 2-Fragment-Fraktur beschrieben [22]. Als Therapiealternative existiert für A3-Frakturen die intramedulläre Marknagelung. In einer biomechanischen Studie konnte bei A3-Frakturen durch intramedulläre Marknagelung eine 
stabilere Reposition erreicht werden, als mit der winkelstabilen Plattenosteosynthese [69]. Zhu et al. konnten in einer randomisierten prospektiven Studie für 2-Fragment-Frakuren eine signifikant höhere Komplikationsrate für die Plattenosteosynthese (31\%) als für die intramedulläre Marknagelung (4\%) zeigen. Trotz einer längeren Rehabilitationszeit nach intramedullärer Marknagelung, waren die funktionellen Endergebnisse vergleichbar. Der Einfluss einer Osteoporose wurde nicht untersucht [50]. Ob die intramedulläre Marknagelung die hohe Komplikationsrate auch im osteoporotischen Knochen senken kann, bleibt unklar. Dies sollte durch randomisierte kontrollierte Studien untersucht werden, die eine Osteoporosediagnostik nach internationalem Standard mit einschließen.

Möglicherweise stellt auch die Augmentation der Schraubenspitzen mittels Knochenzement eine Möglichkeit dar, die Stabilität des Osteosynthesekonstrukts zu erhöhen und sekundäre Dislokationen und Revisionsoperationen zu vermeiden [70].

Als Therapieoption für dislozierte 3- und 4-Fragmentfrakturen hat sich die Primärtherapie mit Hemiprothese oder inverser Schulterprothese etabliert [71]. Ockert et al. beschrieben in einer Matched-Pair Analyse mit 24 Patienten mit dislozierter 3-, oder 4-Fragmentfraktur ähnliche funktionelle Ergebnisse nach einem Jahr bei primärer Versorgung mit inverser Prothese bei deutlich geringerer Komplikationsrate als nach winkelstabiler Plattenosteosynthese [37]. Randomisierte kontrollierte Studien sind nötig, um im osteoporotischen Knochen die Therapieverfahren primäre inverse Schulterprothese und winkelstabile Plattenosteosynthese hinsichtlich funktionellem Ergebnis und Komplikationen zu vergleichen. 


\subsection{Limitationen der Studie}

In der vorliegenden Studie wurde das Auftreten einer sekundären Dislokation nur im Zusammenhang mit dem Vorhandensein einer Osteoporose untersucht. Weitere Risikofaktoren, die eine sekundäre Dislokation begünstigen wurden nicht erfasst. Durch die Ein- und Ausschlusskriterien wurden die Störgrößen verminderte kognitive oder koordinative Fähigkeit, Incompliance oder Unvermögen an einer strukturierten Nachbehandlung teilzunehmen ausgeschlossen. Eine Korrelation der sekundären Dislokation zu anderen potentiellen Risikofaktoren erfolgte jedoch nicht

Weiterhin wurden Patienten gemäß der gängigen Leitlinien anhand des T-Werts der DXA Messung entweder in die Osteoporosegruppe oder Kontrollgruppe eingeteilt. Eine weitere Untersuchung der Auswirkungen des Schweregrads der Osteoporose oder einer Osteopenie wurde nicht durchgeführt. Dieses ist auf eine fehlende einheitliche, akzeptierte Klassifikation zurückzuführen.

Für einzelne Frakturtypen gemäß AO/OTA Klassifikation waren die Fallzahlen sehr gering und in Folge dessen auch die Rate sekundärer Dislokationen und Revision statistisch nicht aussagekräftig. Ob mit höheren Fallzahlen ein Einfluss von Osteoporose auf die Frakturmorphologie nachgewiesen werden kann, bleibt offen und sollte in nachfolgenden Studien mit größeren Fallzahlen untersucht werden.

Es wurde lediglich die winkelstabile Plattenosteosynthese als Therapieverfahren untersucht. Um Vorteile anderer Therapieverfahren bei bestimmten Frakturtypen und Patientencharakteristika mit ausreichend wissenschaftlicher Evidenz herauszuarbeiten, sind weitere randomisierte kontrollierte Studien notwendig. 


\section{Zusammenfassung}

Die Therapie der proximalen Humerusfraktur ist kontrovers diskutiert und stellt vor allem im älteren Patienten mit Osteoporose eine Herausforderung für den behandelnden Arzt dar. Als Verfahren hat sich die winkelstabile Plattenosteosynthese etabliert, die bei guten funktionellen Ergebnissen dennoch eine hohe Komplikationsrate aufweist. Als Risikofaktoren für das Auftreten von Komplikationen wurden in anderen Studien Alter, Rauchen, fehlende anatomische Reposition, fehlende mediale Abstützung und bestimmte Frakturtypen identifiziert. Der Einfluss der Osteoporose wurde in diesem Zusammenhang nicht abschließend geklärt. Ziel war es daher, den Einfluss der Osteoporose auf den Frakturtyp, die Rate der sekundären Dislokation nach winkelstabiler Plattenosteosynthese und die Rate sowie Art der nötigen Revisionsoperationen nach winkelstabiler Plattenosteosynthese der proximalen Humerusfraktur zu untersuchen.

In der beschriebenen Studie wurden über einen Zeitraum von 10 Jahren Patienten über 50 Jahren (Frauen), bzw. über 60 Jahren (Männer) erfasst, die aufgrund einer proximaler Humerusfraktur mittels winkelstabile Plattenosteosynthese versorgt worden waren. Einschlusskriterien waren eine Knochendichtebestimmung mittels DXA, sowie mindestens ein Follow-up von 24 Monaten. Die Frakturklassifikation erfolgte mittels konventioneller Röntgendiagnostik und CT Untersuchungen. Es wurde die Frakturklassifikation nach AO/OTA, die Rate sekundärer Dislokationen sowie Revisionsrate und -verfahren erfasst. Die Auswertungen fanden retrospektiv anhand einer prospektiv geführten Datenbank statt.

Insgesamt konnten 190 Patienten mit einem Durchschnittsalter von $74,3( \pm 9,9)$ Jahren eingeschlossen werden. Hiervon waren 164 weiblich (86,3\%) und 26 männlich (13.7\%). Bei vergleichbaren Anteilen in beiden Gruppen hinsichtlich Alter, Geschlechtsverteilung und anatomischer Reposition, war in der Osteoporosegruppe eine sekundäre Dislokation signifikant häufiger $(24,4 \%)$ als in der Knochenstoffwechsel gesunden Kontrollgruppe (9,5\%). Dabei führten nicht impaktierte Frakturtypen (A3-, B2-, C2-, B3-, und C3) bei Patienten mit Osteoporose signifikant häufiger zu einer sekundären Dislokation $(32,2 \%)$ als bei Patienten ohne Osteoporose (15,4\%). Die Revisionsrate war in der Osteoporosegruppe signifikant höher (18,9\%), als in der Kontrollgruppe (4,8\%). Nicht impaktierte Frakturen mussten in der Osteoporosegruppe mit 25,3\% signifikant häufiger revidiert werden als in der Kontrollgruppe (7,7\%). Die Analyse der Frakturmorphologie zeigte keinen signifikanten Unterschied zwischen den Gruppen.

Die proximale Humerusfraktur des osteoporotischen Knochens trägt abhängig von der Frakturmorphologie ein erhöhtes Risiko für sekundäre Dislokationen und 
Revisionsoperationen. Während impaktierte Frakturen beim Patienten mit Osteoporose niedrige Raten an sekundären Dislokationen aufweisen, stellen nicht-impaktierte Frakturtypen für den Patienten mit Osteoporose ein signifikant erhöhtes Risiko dar, eine sekundäre Dislokation zu erleiden. Ferner besteht für Patienten mit Osteoporose ein erhöhtes Risiko, dass aufgrund dieser Dislokation eine Folgeoperation durchgeführt werden muss. Die Kenntnis des Vorliegens einer Osteoporose sollte daher in der Wahl des Therapieverfahrens berücksichtigt werden, um die Komplikation einer sekundären Dislokation oder einer Revisionsoperation zu vermeiden. 


\section{Literaturverzeichnis}

1. WHO, Assessment of fracture risk and its application to screening for postmenopausal osteoporosis. WHO Technical Report Series, 1994. 843.

2. Haasters, F., et al., [Prevalence and management of osteoporosis in trauma surgery : Implementation of national guidelines during inpatient fracture treatment.]. Unfallchirurg, 2014.

3. Haussler, B., et al., Epidemiology, treatment and costs of osteoporosis in Germany--the BoneEVA Study. Osteoporos Int, 2007. 18(1): p. 77-84.

4. Hadji, P., et al., The epidemiology of osteoporosis--Bone Evaluation Study (BEST): an analysis of routine health insurance data. Dtsch Arztebl Int, 2013. 110(4): p. 52-7.

5. Dachverband Osteologie, Prophylaxe, Diagnostik und Therapie der Osteoporose bei Männern ab dem 60. Lebensjahr und bei postmenopausalen Frauen 2014.

6. $\quad$ Court-Brown, C.M. and B. Caesar, Epidemiology of adult fractures: A review. Injury, 2006. 37(8): p. 691-697.

7. Lauritzen, J.B., et al., Changing incidence and residual lifetime risk of common osteoporosis-related fractures. Osteoporos Int, 1993. 3(3): p. 127-32.

8. $\quad$ Kristiansen, B., et al., Epidemiology of proximal humeral fractures. Acta Orthop Scand, 1987. 58(1): p. 75-7.

9. Lind, T., K. Kroner, and J. Jensen, The epidemiology of fractures of the proximal humerus. Arch Orthop Trauma Surg, 1989. 108(5): p. 285-7.

10. Court-Brown, C.M., A. Garg, and M.M. McQueen, The translated two-part fracture of the proximal humerus. Epidemiology and outcome in the older patient. J Bone Joint Surg Br, 2001. 83(6): p. 799-804.

11. Calvo, E., et al., Nondisplaced proximal humeral fractures: high incidence among outpatient-treated osteoporotic fractures and severe impact on upper extremity function and patient subjective health perception. J Shoulder Elbow Surg, 2011. 20(5): p. 795-801.

12. Rose, S.H., et al., Epidemiologic features of humeral fractures. Clin Orthop Relat Res, 1982(168): p. 24-30.

13. Horak, J. and B.E. Nilsson, Epidemiology of fracture of the upper end of the humerus. Clin Orthop Relat Res, 1975(112): p. 250-3.

14. Palvanen, M., et al., Update in the epidemiology of proximal humeral fractures. Clin Orthop Relat Res, 2006. 442: $\mathrm{p}$. 87-92.

15. Kannus, P., et al., Rate of proximal humeral fractures in older Finnish women between 1970 and 2007. Bone, 2009. 44(4): p. 656-9.

16. Bell, J.E., et al., Trends and variation in incidence, surgical treatment, and repeat surgery of proximal humeral fractures in the elderly. J Bone Joint Surg Am, 2011. 93(2): p. 121-31.

17. Maravic, M., et al., Incidence and cost of osteoporotic fractures in France during 2001. A methodological approach by the national hospital database. Osteoporos Int, 2005. 16(12): p. 1475-80.

18. Palvanen, M., et al., The injury mechanisms of osteoporotic upper extremity fractures among older adults: a controlled study of 287 consecutive patients and their 108 controls. Osteoporos Int, 2000. 11(10): p. 822-31.

19. Clement, N.D., et al., Multiple fractures in the elderly. J Bone Joint Surg Br, 2012. 94(2): p. 231-6.

20. Roux, A., et al., Epidemiology of proximal humerus fractures managed in a trauma center. Orthop Traumatol Surg Res, 2012. 98(6): p. 715-9.

21. Codman, E.A., Fractures in relation to the subacromial bursa. The Shoulder, 1934.

22. Neer, C.n., Displaced proximal humeral fractures. Part I. Classification and evaluation. J Bone Joint Surg Am, 1970. 52(6): p. 1077-1089.

23. Mueller, M., et al., The comprehensive classification of fractures of long bones Berlin: Springer, 1990.

24. Hertel, R., Fractures of the proximal humerus in osteoporotic bone. Osteoporosis international : a journal established as result of cooperation between the European Foundation for Osteoporosis and the National Osteoporosis Foundation of the USA, 2005. 16 Suppl 2: p. S65-72.

25. Majed, A., et al., Proximal humeral fracture classification systems revisited. J Shoulder Elbow Surg, 2011. 20(7): p. 1125-32.

26. Resch, H., et al., Classification of proximal humeral fractures based on a pathomorphologic analysis. Journal of Shoulder and Elbow Surgery, 2015.

27. Lill, H., et al., [Osteoporotic fractures of the proximal humerus]. Chirurg, 2012. 83(10): p. 858-65.

28. Court-Brown, C.M., A. Garg, and M.M. McQueen, The epidemiology of proximal humeral fractures. Acta Orthop Scand, 2001. 72(4): p. 365-71.

29. Marsh, J.L., et al., Fracture and dislocation classification compendium - 2007: Orthopaedic Trauma Association classification, database and outcomes committee. J Orthop Trauma, 2007. 21(10 Suppl): p. S1-133.

30. Hertel, R., et al., Predictors of humeral head ischemia after intracapsular fracture of the proximal humerus. J Shoulder Elbow Surg, 2004. 13(4): p. 427-33.

31. Foruria, A.M., et al., The pattern of the fracture and displacement of the fragments predict the outcome in proximal humeral fractures. J Bone Joint Surg Br, 2011. 93(3): p. 378-86.

32. Krettek, C. and U. Wiebking, [Proximal humerus fracture: is fixed-angle plate osteosynthesis superior to conservative treatment?]. Unfallchirurg, 2011. 114(12): p. 1059-67.

33. Fjalestad, T., et al., Health and cost consequences of surgical versus conservative treatment for a comminuted proximal humeral fracture in elderly patients. Injury, 2010. 41(6): p. 599-605.

34.

Fjalestad, T., et al., Surgical treatment with an angular stable plate for complex displaced proximal humeral fractures in elderly patients: a randomized controlled trial. J Orthop Trauma, 2012. 26(2): p. 98-106. 
35. Olerud, P., et al., Internal fixation versus nonoperative treatment of displaced 3-part proximal humeral fractures in elderly patients: a randomized controlled trial. J Shoulder Elbow Surg, 2011. 20(5): p. 747-55.

36. Handoll, H.H. and S. Brorson, Interventions for treating proximal humeral fractures in adults. Cochrane Database Syst Rev, 2015(11): p. Cd000434.

37. Ockert, B., et al., [Reverse shoulder arthroplasty for primary fracture treatment. Displaced three and four part fractures of the proximal humerus in the elderly patient]. Unfallchirurg, 2013. 116(8): p. 684-90.

38. Solberg, B.D., et al., Locked plating of 3- and 4-part proximal humerus fractures in older patients: the effect of initial fracture pattern on outcome. J Orthop Trauma, 2009. 23(2): p. 113-9.

39. Krappinger, D., et al., Predicting failure after surgical fixation of proximal humerus fractures. Injury, 2011. 42(11): p. $1283-8$.

40. Roderer, G., et al., Clinical results for minimally invasive locked plating of proximal humerus fractures. J Orthop Trauma, 2010. 24(7): p. 400-6.

41. Ockert, B., et al., Long-term functional outcomes (median 10 years) after locked plating for displaced fractures of the proximal humerus. J Shoulder Elbow Surg, 2014.

42. Sudkamp, N., et al., Open reduction and internal fixation of proximal humeral fractures with use of the locking proximal humerus plate. Results of a prospective, multicenter, observational study. J Bone Joint Surg Am, 2009. 91(6): p. 1320-8.

43. Thanasas, C., et al., Treatment of proximal humerus fractures with locking plates: a systematic review. J Shoulder Elbow Surg, 2009. 18(6): p. 837-44.

44. Chudik, S.C., P. Weinhold, and L.E. Dahners, Fixed-angle plate fixation in simulated fractures of the proximal humerus: a biomechanical study of a new device. J Shoulder Elbow Surg, 2003. 12(6): p. 578-88.

45. Siwach, R., et al., Internal fixation of proximal humeral fractures with locking proximal humeral plate (LPHP) in elderly patients with osteoporosis. J Orthop Traumatol, 2008. 9(3): p. 149-53.

46. Vallier, H.A., Treatment of proximal humerus fractures. J Orthop Trauma, 2007. 21(7): p. 469-76.

47. Sproul, R.C., et al., A systematic review of locking plate fixation of proximal humerus fractures. Injury, 2011. 42(4): $\mathrm{p}$. 408-13.

48. Owsley, K.C. and J.T. Gorczyca, Fracture displacement and screw cutout after open reduction and locked plate fixation of proximal humeral fractures [corrected]. J Bone Joint Surg Am, 2008. 90(2): p. 233-40.

49. Hardeman, F., et al., Predictive factors for functional outcome and failure in angular stable osteosynthesis of the proximal humerus. Injury, 2012. 43(2): p. 153-8.

50. Zhu, Y., et al., Locking intramedullary nails and locking plates in the treatment of two-part proximal humeral surgical neck fractures: a prospective randomized trial with a minimum of three years of follow-up. J Bone Joint Surg Am, 2011. 93(2): p. 159-68.

51. Sudkamp, N.P., et al., Path analysis of factors for functional outcome at one year in 463 proximal humeral fractures. J Shoulder Elbow Surg, 2011. 20(8): p. 1207-16.

52. Haasters, F., et al., Complications of locked plating for proximal humeral fractures-are we getting any better? J Shoulder Elbow Surg, 2016. 25(10): p. e295-303.

53. Spross, C., et al., The PHILOS plate for proximal humeral fractures--risk factors for complications at one year. J Trauma Acute Care Surg, 2012. 72(3): p. 783-92.

54. Tingart, M.J., et al., Proximal humeral fractures: regional differences in bone mineral density of the humeral head affect the fixation strength of cancellous screws. J Shoulder Elbow Surg, 2006. 15(5): p. 620-4.

55. Goldhahn, J., et al., Influence of osteoporosis on fracture fixation--a systematic literature review. Osteoporos Int, 2008. 19(6): p. 761-72.

56. Hepp, P., et al., Bone quality measured by the radiogrammetric parameter "cortical index" and reoperations after locking plate osteosynthesis in patients sustaining proximal humerus fractures. Arch Orthop Trauma Surg, 2009. 129(9): p. 1251-9.

57. Siebenrock, K.A. and C. Gerber, The reproducibility of classification of fractures of the proximal end of the humerus. J Bone Joint Surg Am, 1993. 75(12): p. 1751-5.

58. Grampp, S., et al., Leitlinien zur Anwendung densitometischer Verfahren in der Postmenopause. Journal für Mineralstoffwechsel, 2004. 11(2): p. 7-10.

59. Grampp, S., et al., Leitlinien zur Anwendung densitometrischer Verfahren. Journal für Mineralstoffwechsel, 2001. 8(2): p. 50-51.

60. World Medical Association Declaration of Helsinki: ethical principles for medical research involving human subjects. Jama, 2013. 310(20): p. 2191-4.

61. Hirzinger, C., M. Tauber, and H. Resch, [Proximal humerus fracture: new aspects in epidemiology, fracture morphology, and diagnostics]. Unfallchirurg, 2011. 114(12): p. 1051-8.

62. Solberg, B.D., et al., Surgical treatment of three and four-part proximal humeral fractures. J Bone Joint Surg Am, 2009. $91(7)$ : p. 1689-97.

63. Fjalestad, T., et al., Fractures in the proximal humerus: functional outcome and evaluation of 70 patients treated in hospital. Arch Orthop Trauma Surg, 2005. 125(5): p. 310-6.

64. Hepp, P., et al., The surgical approach for locking plate osteosynthesis of displaced proximal humeral fractures influences the functional outcome. J Shoulder Elbow Surg, 2008. 17(1): p. 21-8.

65. Brunner, F., et al., Open reduction and internal fixation of proximal humerus fractures using a proximal humeral locked plate: a prospective multicenter analysis. J Orthop Trauma, 2009. 23(3): p. 163-72.

66. Lee, C.W. and S.J. Shin, Prognostic factors for unstable proximal humeral fractures treated with locking-plate fixation. J Shoulder Elbow Surg, 2009. 18(1): p. 83-8.

67. Jung, S.W., et al., Factors that Influence Reduction Loss in Proximal Humerus Fracture Surgery. J Orthop Trauma, 2015. 29(6): p. 276-82.

68. Roderer, G., et al., Second generation locked plating of proximal humerus fractures--a prospective multicentre observational study. Int Orthop, 2011. 35(3): p. 425-32.

69. Yoon, R.S., et al., A comprehensive update on current fixation options for two-part proximal humerus fractures: a biomechanical investigation. Injury, 2014. 45(3): p. 510-4.

70. Schliemann, B., et al., How to enhance the stability of locking plate fixation of proximal humerus fractures? An overview of current biomechanical and clinical data. Injury, 2015. 46(7): p. 1207-14.

71. Gallinet, D., et al., Three or four parts complex proximal humerus fractures: hemiarthroplasty versus reverse prosthesis: a comparative study of 40 cases. Orthop Traumatol Surg Res, 2009. 95(1): p. 48-55. 


\section{Danksagung}

Zunächst möchte ich meine große Dankbarkeit an PD Dr. med. Florian Haasters ausdrücken, der mir die Arbeit an diesem Projekt für meine Dissertation ermöglicht und mich stets in allen Fragen exzellent beraten hat.

Weiterhin möchte ich allen beteiligten Mitarbeitern der Klinik für Allgemeine-, Unfall- und Wiederherstellungschirurgie der Ludwig-Maximilians-Universität München für die gute Zusammenarbeit bedanken.

Besonderer Dank gebührt meinen Eltern Angelika und Siegbert für die Ermöglichung des Medizinstudiums und dieser Arbeit.

Zum Schluss möchte ich Jana danken für ihre tagtägliche Unterstützung und ihr geduldiges Zuhören, was für die Vollendung dieser Arbeit essentiell war. 


\section{Eidesstattliche Versicherung}

Kindsvater, Johannes

Name, Vorname

Ich erkläre hiermit an Eides statt,

dass ich die vorliegende Dissertation mit dem Thema

Osteoporose als Risikofaktor für das Versagen der winkelstabilen Plattenosteosynthese am proximalen Humerus

selbständig verfasst, mich außer der angegebenen keiner weiteren Hilfsmittel bedient und alle Erkenntnisse, die aus dem Schrifttum ganz oder annähernd übernommen sind, als solche kenntlich gemacht und nach ihrer Herkunft unter Bezeichnung der Fundstelle einzeln nachgewiesen habe.

Ich erkläre des Weiteren, dass die hier vorgelegte Dissertation nicht in gleicher oder in ähnlicher Form bei einer anderen Stelle zur Erlangung eines akademischen Grades eingereicht wurde.

Augsburg, 23.06.2018

Johannes Kindsvater 